\title{
ASYMPTOTIC EIGENVALUE MOMENTS FOR LINEAR MULTIUSER DETECTION*
}

\author{
LINBO LI ${ }^{\dagger}$, ANTONIA M. TULINO ${ }^{\dagger}$, AND SERGIO VERDÚ ${ }^{\dagger}$
}

\begin{abstract}
Using the theory of lattices of non-crossing partitions, an explicit expression for the moments and free cumulants of the asymptotic distribution of certain infinite random matrices is obtained and extended to several cases. From the explicit expression, we give a self-contained proof of the Tse-Hanly formula for the output signal-to-interference-plus-noise ratio of MMSE multiuser detector. We use the moment results to design an asymptotic reduced-rank linear multiuser detector. Also we apply these results to analyze linear-conjugate MMSE receivers and multiple antenna receivers.
\end{abstract}

1. Introduction. In order to gain insight into the performance of receivers in a DS-CDMA system with large processing gain and many users, much work has been devoted to the asymptotic analysis for synchronous DS-CDMA with random spreading $[17,7,15]$. In [15], the asymptotic output signal-to-interference-plus-noise ratio (SINR) of the MMSE receiver is shown to satisfy a fixed point equation by using recent results on the asymptotic eigenvalue distribution of certain infinite random matrices. The reduced-rank MMSE receiver is proposed and analyzed in [10]. As discussed in [10] the moments of the asymptotic eigenvalue distribution of some infinite random matrix are relevant to find the large system limit of the output SINR and the optimal weights of the reduced-rank receiver. Several recursive procedures to obtain the asymptotic eigenvalue moments are proposed in [20,10]. A certain combinatorial problem is shown in [20] to be equivalent to the problem of computing the moments explicitly.

In Section 2, using the theory of lattices of non-crossing partitions, explicit expressions for the asymptotic eigenvalue moments of the correlation matrix of the CDMA signal $\mathbf{S D S}^{H}$ ( $\mathbf{S}$ is a matrix whose columns are the normalized spreading sequences of the users, and $\mathbf{D}$ is a diagonal matrix with diagonal elements the received powers of the users), with $(\cdot)^{H}$ denoting the conjugate transpose, are obtained for several classes of infinite random matrices. In case (i) $\mathbf{S}$ consists of i.i.d. random variables, and the explicit expression for the moments is obtained by solving the combinatorial problem formulated in [20]. This kind of $\mathbf{S}$ (i.i.d. entries) arises when we consider a single receive antenna DS-CDMA system with random spreading. In case (ii) each column of $\mathbf{S}$ consists of repeated i.i.d. sequences with block i.i.d. phases; this kind of $\mathbf{S}$ arises when we consider multiple receive antenna randomly-spread DS-CDMA where the phases of the fading coefficients at different antenna elements are independent. In case (iii) each column of $\mathbf{S}$ consists of repeated i.i.d. sequences with linearly

\footnotetext{
*Invited paper; received on April 16, 2001, accepted for publication on August 21, 2001.

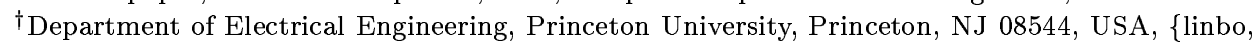
atulino,verdu\}@ee.princeton.edu
} 
increasing phases; this kind of $\mathbf{S}$ arises when we consider multiantenna receiver for DS-CDMA in line-of-sight transmission. In cases (iv), (v) and (vi), each column of $\mathbf{S}$ is formed by stacking the matrices in (i), (ii) and (iii) with their conjugate. These kinds of $\mathbf{S}$ arise when we consider the newly proposed linear-conjugate MMSE (LCM) receiver [16] for single antenna and multiple antenna systems.

There are several motivations and applications for the results in Section 2, which are discussed in detail in Section 3. In Section 3.1, the large-system limit of the optimal weights for the reduced-rank MMSE/LCM receivers is obtained from the explicit expression of the moments. Six environments are considered, corresponding to the direct applications of the results of the six classes of matrices obtained in Section 2. Receivers that employ the asymptotic limit of the optimal weights instead of the optimal weights themselves are called asymptotic reduced-rank receivers. The desirable feature of these receivers is that the asymptotic limit of the optimal weights does not depend on the realizations of the spreading sequences, which is particularly useful when the CDMA system uses long sequences. With the pre-calculated asymptotic weights, the reduced-rank MMSE/LCM receivers can be implemented in an efficient way as discussed in [12]. In Section 3.2 the asymptotic performance of the receivers considered in 3.1 is given and the asymptotic equivalence of them is established. In Section 4, based on the result on the moments we give a self-contained proof of the fixed point equation satisfied by the asymptotic output SINR of the MMSE receiver, which is originally derived in [15] by means of the Silverstein and Bai theorem [13]. Section 5 contains our numerical results, which show that for reasonably large processing gain $(N=32)$, the output SINR of the asymptotic reduced-rank receiver lies within $1 \mathrm{~dB}$ from that of the reduced-rank receiver using spreadingsequence-dependent optimal weights. For larger processing gain, say 80 or larger, the performance gap between the two receivers is less than $0.5 \mathrm{~dB}$.

2. Asymptotic Eigenvalue Moments. In this section we state our results on the asymptotic eigenvalue moments. Corresponding situations in which these results can be applied are discussed in Section 3.

Definition. Let us consider the vector space of the $n \times n$ matrices $\mathcal{M}_{n}(C)$ whose elements are complex random variables. $\mathcal{M}_{n}(C)$ with the ordinary sum, and product, and adjunction (Hermitian transpose) is a $*$-algebra. It is said that the random matrix $\mathbf{V}_{n} \in \mathcal{M}_{n}(C)$ has the $m$-th limit moment $\lambda_{m}$ if there exists finite limit

$$
\lambda_{m} \triangleq \lim _{n \rightarrow \infty} \frac{1}{n} \mathrm{E}\left[\operatorname{Trace}\left\{\mathbf{V}_{n}^{m}\right\}\right] .
$$

The set of all limit moments of the matrix $\mathbf{V}_{n}$ defines the the limit distribution of $\mathbf{V}_{n}$.

If $\mathbf{V}_{n}$ is a self-adjoint matrix, the limit distribution of $\mathbf{V}_{n}$ is the function, $F(\lambda)$, described by the collection of its moments $[18,9]$ as follow:

$$
\int \lambda^{m} d F(\lambda) \triangleq \lim _{n \rightarrow \infty} \frac{1}{n} \mathrm{E}\left[\operatorname{Trace}\left\{\mathbf{V}_{n}^{m}\right\}\right]
$$


$F(\lambda)$ is also called the limit eigenvalue distribution of $\mathbf{V}_{n}$ and it is easy to see $[18,9]$ that:

$$
F(\lambda)=\lim _{n \rightarrow \infty} E\left[F_{n}(\lambda)\right]
$$

where $F_{n}(\lambda)$ is the empirical distribution of the eigenvalues of $\mathbf{V}_{n}$ [18].

Let us introduce here the necessary notation used in the following results: suppose a vector of $k$ integers $m_{1}, \ldots, m_{k}$ is partitioned into $n$ equivalence classes under the equivalence relation $a=b$, and the cardinalities of the equivalence classes are given by $f_{1}, \ldots, f_{n}$, then we define the following function:

$$
f\left(m_{1}, \ldots, m_{k}\right) \triangleq f_{1} ! \cdots f_{n} !
$$

For example, $f(1,1,4,2,1,2)=3 ! \cdot 2 ! \cdot 1 !$.

The proofs of Propositions 1 and 4 are given in Appendices A and B respectively. Propositions 2, 3, 5, 6 can be shown following similar methods used in the proof of Proposition 4 and a brief explanation of the proofs is given at the end of Appendix B.

\subsection{I.I.D. Sequences.}

Proposition 1. Suppose the $N \times K$ matrix $\mathbf{S}$ consists of i.i.d. zero-mean random variables with variance $1 / N, \mathbf{D}$ is a $K \times K$ diagonal matrix with non-negative diagonal elements, and the empirical distribution of the diagonal elements of $\mathbf{D}$ converges almost surely (a.s.) to a non-random limit distribution. Then the mth moment of the limit eigenvalue distribution $G(\lambda)$ of $\mathbf{S D S}^{H}$ as $K, N$ go to infinity with $K / N=\beta$ is:

$$
\int \lambda^{m} d G(\lambda)=\sum_{k=1}^{m} \beta^{k} \sum_{m_{1}+\cdots+m_{k}=m} c\left(m_{1}, \ldots, m_{k}\right) \cdot \mathrm{E}\left[\Lambda^{m_{1}}\right] \cdots \mathrm{E}\left[\Lambda^{m_{k}}\right]
$$

where $\Lambda$ is a nonnegative random variable whose distribution is the non-random limit distribution of the diagonal elements of $\mathbf{D}$, and

$$
c\left(m_{1}, \ldots, m_{k}\right)=\frac{m !}{(m-k+1) ! \cdot f\left(m_{1}, \ldots, m_{k}\right)},
$$

with $f\left(m_{1}, \ldots, m_{k}\right)$ as defined above.

Proof. In Appendix A.

An equivalent result on the asymptotic eigenvalue moments in the i.i.d.-sequences case can be found in [21] in a slightly different form. The reason we still present our proof for this case (in Appendix A) is to introduce necessary techniques that are to be used in the generalization to other cases. A few remarks on the assumption made in Proposition 1 will be made in Section 3.1.1. 


\subsection{Repeated Sequences with Block I.I.D. Phases.}

Proposition 2. Let $N=L N^{\prime}$. Let $\mathbf{t}_{k}=\left(U_{k, 1}, \ldots, U_{k, N^{\prime}}\right)^{T}(1 \leq k \leq K)$, where $U_{k, j}$ 's $\left(1 \leq k \leq K, 1 \leq j \leq N^{\prime}\right)$ are an array of real- valued i.i.d. zero-mean random variables with unit variance. Let $\mathbf{S}=\left(\mathbf{s}_{1}, \ldots, \mathbf{s}_{K}\right)$ where

$$
\mathbf{s}_{k}=\frac{1}{\sqrt{N}}\left(\mathbf{t}_{k}^{T} \mathrm{e}^{j \phi_{k, 1}}, \ldots, \mathbf{t}_{k}^{T} \mathrm{e}^{j \phi_{k, L}}\right)^{T},
$$

and $\phi_{k, l}$ 's $(1 \leq k \leq K, 1 \leq l \leq L)$ are i.i.d. random variables uniformly distributed on $[0,2 \pi)$, and $1 / \sqrt{N}$ is the normalizing factor. $\mathbf{D}$ is a $K \times K$ diagonal matrix with nonnegative diagonal elements, and the empirical distribution of the diagonal elements of $\mathbf{D}$ converges a.s. to a non-random limit distribution. Then the mth moment of the limit eigenvalue distribution $G(\lambda)$ of $\mathbf{S D S}^{H}$ as $K, N$ go to infinity with $K / N=\beta$ is the same as in Proposition 1, i.e. (1).

Proof. Refer to Appendix B for a brief explanation of the proof.

\subsection{Conjugate Sequences.}

Proposition 3. Let $N=2 N^{\prime}$ '. Let $\mathbf{t}_{k}=\left(U_{k, 1}, \ldots, U_{k, N^{\prime}}\right)^{T}(1 \leq k \leq K)$, where $U_{k, j}$ 's $\left(1 \leq k \leq K, 1 \leq j \leq N^{\prime}\right)$ are an array of real- valued i.i.d. zero-mean random variables with unit variance. Let $\mathbf{S}=\left(\mathbf{s}_{1}, \ldots, \mathbf{s}_{K}\right)$ where

$$
\mathbf{s}_{k}=\frac{1}{\sqrt{N}}\left(\mathbf{t}_{k}^{T} \mathrm{e}^{j \phi_{k}}, \mathbf{t}_{k}^{T} \mathrm{e}^{-j \phi_{k}}\right)^{T},
$$

and $\phi_{k}$ 's $(1 \leq k \leq K)$ are i.i.d. random variables uniformly distributed on $[0,2 \pi)$, and $1 / \sqrt{N}$ is the normalizing factor. $\mathbf{D}$ is a $K \times K$ diagonal matrix with nonnegative diagonal elements, and the empirical distribution of the diagonal elements of D converges a.s. to a non-random limit distribution. Then the mth moment of the limit eigenvalue distribution $G(\lambda)$ of $\mathbf{S D S}^{H}$ as $K, N$ go to infinity with $K / N=\beta$ is the same as in Proposition 1.

Proof. Refer to Appendix B for a brief explanation of the proof.

\subsection{Repeated Sequences with Conjugate Block I.I.D. Phases.}

Proposition 4. Let $N=2 L N^{\prime}$. Let $\mathbf{t}_{k}=\left(U_{k, 1}, \ldots, U_{k, N^{\prime}}\right)^{T}(1 \leq k \leq K)$, where $U_{k, j}$ 's $\left(1 \leq k \leq K, 1 \leq j \leq N^{\prime}\right)$ are an array of real- valued i.i.d. zero-mean random variables with unit variance. Let $\mathbf{S}=\left(\mathbf{s}_{1}, \ldots, \mathbf{s}_{K}\right)$ where

$$
\mathbf{s}_{k}=\frac{1}{\sqrt{N}}\left(\mathbf{t}_{k}^{T} \mathrm{e}^{j \phi_{k, 1}}, \ldots, \mathbf{t}_{k}^{T} \mathrm{e}^{j \phi_{k, L}}, \mathbf{t}_{k}^{T} \mathrm{e}^{-j \phi_{k, 1}}, \ldots, \mathbf{t}_{k}^{T} \mathrm{e}^{-j \phi_{k, L}}\right)^{T},
$$

and $\phi_{k, l}$ 's $(1 \leq k \leq K, 1 \leq l \leq L)$ are i.i.d. random variables uniformly distributed on $[0,2 \pi)$, and $1 / \sqrt{N}$ is the normalizing factor. $\mathbf{D}$ is a $K \times K$ diagonal matrix with nonnegative diagonal elements, and the empirical distribution of the diagonal elements of 
D converges a.s. to a non-random limit distribution. Then the mth moment of the limit eigenvalue distribution $G(\lambda)$ of $\mathbf{S D S}^{H}$ as $K, N$ go to infinity with $K / N=\beta$ is the same as in Proposition 1.

Proof. In Appendix B.

\subsection{Repeated Sequences with Linearly Increasing Phases.}

Proposition 5. Let $N=L N^{\prime}$. Let $\mathbf{t}_{k}=\left(U_{k, 1}, \ldots, U_{k, N^{\prime}}\right)^{T}(1 \leq k \leq K)$, where $U_{k, j}$ 's $\left(1 \leq k \leq K, 1 \leq j \leq N^{\prime}\right)$ are an array of real- valued i.i.d. zero-mean random variables with unit variance. Let $\mathbf{S}=\left(\mathbf{s}_{1}, \ldots, \mathbf{s}_{K}\right)$ where

$$
\mathbf{s}_{k}=\frac{1}{\sqrt{N}}\left(\mathbf{t}_{k}^{T} \mathrm{e}^{j \phi_{k}}, \mathbf{t}_{k}^{T} \mathrm{e}^{2 j \phi_{k}}, \ldots, \mathbf{t}_{k}^{T} \mathrm{e}^{L j \phi_{k}}\right)^{T},
$$

and $\phi_{k}$ 's $(1 \leq k \leq K)$ are i.i.d. random variables uniformly distributed on $[0,2 \pi)$, and $1 / \sqrt{N}$ is the normalizing factor. $\mathbf{D}$ is a $K \times K$ diagonal matrix with nonnegative diagonal elements, and the empirical distribution of the diagonal elements of D converges a.s. to a non-random limit distribution. Then the mth moment of the limit eigenvalue distribution $G(\lambda)$ of $\mathbf{S D S}^{H}$ as $K, N$ go to infinity with $K / N=\beta$ is the same as in Proposition 1.

Proof. Refer to Appendix B for a brief explanation of the proof.

\subsection{Repeated Sequences with Conjugate Linearly Increasing Phases.}

Proposition 6. Let $N=2 L N^{\prime}$. Let $\mathbf{t}_{k}=\left(U_{k, 1}, \ldots, U_{k, N^{\prime}}\right)^{T}(1 \leq k \leq K)$, where $U_{k, j}$ 's $\left(1 \leq k \leq K, 1 \leq j \leq N^{\prime}\right)$ are an array of real- valued i.i.d. zero-mean random variables with unit variance. Let $\mathbf{S}=\left(\mathbf{s}_{1}, \ldots, \mathbf{s}_{K}\right)$ where

$$
\mathbf{s}_{k}=\frac{1}{\sqrt{N}}\left(\mathbf{t}_{k}^{T} \mathrm{e}^{j \phi_{k}}, \ldots, \mathbf{t}_{k}^{T} \mathrm{e}^{L j \phi_{k}}, \mathbf{t}_{k}^{T} \mathrm{e}^{-j \phi_{k}}, \ldots, \mathbf{t}_{k}^{T} \mathrm{e}^{-L j \phi_{k}}\right)^{T},
$$

and $\phi_{k}$ 's $(1 \leq k \leq K)$ are i.i.d. random variables uniformly distributed on $[0,2 \pi)$, and $1 / \sqrt{N}$ is the normalizing factor. $\mathbf{D}$ is a $K \times K$ diagonal matrix with nonnegative diagonal elements, and the empirical distribution of the diagonal elements of D converges a.s. to a non-random limit distribution. Then the mth moment of the limit eigenvalue distribution $G(\lambda)$ of $\mathbf{S D S}^{H}$ as $K, N$ go to infinity with $K / N=\beta$ is the same as in Proposition 1.

Proof. Refer to Appendix B for a brief explanation of the proof.

3. Motivations and Applications. In this section, we discuss motivations and applications of the results in Section 2.

3.1. Asymptotic Reduced-Rank Receiver. Expressions for the asymptotic moments can be used to implement the asymptotic reduced-rank MMSE/LCM receivers. With the pre-calculated asymptotic weights, the reduced-rank MMSE/LCM 
receivers can be implemented in an efficient way as discussed in [12]. In what follows, we consider six situations: (i) reduced-rank single antenna MMSE receiver; (ii) reduced-rank multiantenna MMSE receiver; (iii) reduced-rank single antenna LCM receiver; (iv) reduced-rank multiantenna LCM receiver; (v) reduced-rank multiantenna MMSE receiver in line-of-sight transmission; (vi) reduced-rank multiantenna LCM receiver in line-of-sight transmission. These situations correspond to applications of Propositions 1-6, respectively.

In these situations, we assume synchronous DS-CDMA with processing gain $N$ and number of users $K . A_{k}$ is the received amplitude of user $k, b_{k} \in\{ \pm 1\}$ is the bit transmitted by user $k$, and $\mathbf{s}_{k}$ is the spreading sequence of user $k$. For a single antenna receiver, the phase of the complex fading coefficient of user $k$ is $\mathrm{e}^{j \phi_{k}}$. For a multiantenna receiver, the phase of the complex fading coefficient of user $k$ at the $l$ th antenna element is $\mathrm{e}^{j \phi_{k}(l)}$. The phases $\left(\phi_{k}\right.$ 's or $\phi_{k}(l)$ 's) are modelled as i.i.d. random variables uniformly distributed on $[0,2 \pi)$. The spreading sequences are assumed known at the receiver. Perfect knowledge of the fading channel gain (magnitude and phase) at the receiver is also assumed, which could be the case at a base station. For a single antenna receiver, the noise vector at the output of the chipmatched filter is $\mathbf{n}$. For a multiantenna receiver, $\mathbf{n}_{l}$ is the chip-matched filter output noise vector at the $l$ th antenna element, and they are independent across antenna elements. The distribution of the noise vector is $\mathcal{N}\left(\mathbf{0}, \sigma^{2} \mathbf{I}\right)$. Random spreading is used to model the spreading sequences. Specifically,

$$
\mathbf{s}_{k}=\frac{1}{\sqrt{N}} \cdot\left(V_{k, 1}, \ldots, V_{k, N}\right)^{T}
$$

where $V_{k, j}$ 's $(1 \leq k \leq K, 1 \leq j \leq N)$ are i.i.d. random variables taking equiprobable values on $\{ \pm 1\} .1 / \sqrt{N}$ normalizes the energy of the spreading sequences.

3.1.1. Reduced-Rank Single Antenna MMSE Receiver. In a single antenna DS-CDMA system, the output of the chip-matched filter is

$$
\mathbf{r}=\sum_{k=1}^{K} A_{k} \mathrm{e}^{j \phi_{k}} b_{k} \mathbf{s}_{k}+\mathbf{n}
$$

where $A_{k} \mathrm{e}^{j \phi_{k}}$ is a complex gain taking into account the channel effects.

Put in matrix form

$$
\mathbf{r}=\mathbf{S A b}+\mathbf{n},
$$

where $\mathbf{S}=\left(\mathbf{s}_{1} \mathrm{e}^{j \phi_{1}}, \ldots, \mathbf{s}_{K} \mathrm{e}^{j \phi_{K}}\right)$ is an $N \times K$ matrix, $\mathbf{A}=\operatorname{diag}\left\{A_{1}, \ldots, A_{K}\right\}$, and $\mathbf{b}=\left(b_{1}, \ldots, b_{K}\right)^{T}$. The MMSE receiver for user 1 is

$$
\mathbf{c}=\left(\mathbf{S}_{1} \mathbf{D}_{1} \mathbf{S}_{1}^{H}+\sigma^{2} \mathbf{I}\right)^{-1} \mathbf{S}_{1} \mathrm{e}^{j \phi_{1}},
$$


where $\mathbf{S}_{1}=\left(\mathbf{s}_{2} \mathrm{e}^{j \phi_{2}}, \ldots, \mathbf{s}_{K} \mathrm{e}^{j \phi_{K}}\right)$, and $\mathbf{D}_{1}=\operatorname{diag}\left\{A_{2}^{2}, \ldots, A_{K}^{2}\right\}$. The rank $D(D \leq N)$ reduced-rank MMSE receiver for user 1 is

$$
\mathbf{c}_{D}=\sum_{m=0}^{D-1} w_{m}\left(\mathbf{S}_{1} \mathbf{D}_{1} \mathbf{S}_{1}^{H}+\sigma^{2} \mathbf{I}\right)^{m} \mathbf{s}_{1} \mathrm{e}^{j \phi_{1}}
$$

where the weight vector $\mathbf{w}=\left(w_{0}, \ldots, w_{D-1}\right)^{T}$ is chosen to maximize the output SINR and is given by $[11,16]$

$$
\mathbf{w}=\left[\begin{array}{ccc}
\mathcal{H}_{1}+A_{1}^{2} \mathcal{H}_{0} \mathcal{H}_{0} & \cdots & \mathcal{H}_{D}+A_{1}^{2} \mathcal{H}_{D-1} \mathcal{H}_{0} \\
\vdots & \ddots & \vdots \\
\mathcal{H}_{D}+A_{1}^{2} \mathcal{H}_{D-1} \mathcal{H}_{0} & \cdots & \mathcal{H}_{2 D-1}+A_{1}^{2} \mathcal{H}_{D-1} \mathcal{H}_{D-1}
\end{array}\right]^{-1}\left[\begin{array}{c}
\mathcal{H}_{0} \\
\vdots \\
\mathcal{H}_{D-1}
\end{array}\right]
$$

where the $(i, j)$-entry of the matrix above is $\mathcal{H}_{i+j-1}+A_{1}^{2} \mathcal{H}_{i-1} \mathcal{H}_{j-1}$ with

$$
\mathcal{H}_{m}=\mathbf{s}_{1}^{H}\left(\mathbf{S}_{1} \mathbf{D}_{1} \mathbf{S}_{1}^{H}+\sigma^{2} \mathbf{I}\right)^{m} \mathbf{s}_{1} .
$$

From the expression above, we see that the optimal weights depend on the realizations of the spreading sequences. Therefore in a system using long sequences, they need to be computed and updated symbol to symbol, which hampers real-time implementation. It is therefore desirable to find a set of suboptimal weights that do not depend on the spreading sequences while maintaining acceptable performance. The asymptotic values of the weights in the large system limit seem to be a promising solution. The large system limit is taken as $N$ and $K$ go to infinity with $K / N=\beta$. Using Corollary 1 in [4] and the results in [21], it can be shown that if the empirical distribution of the diagonal elements of the matrix $\mathbf{D}_{1}$ converges almost surely (a.s.) to a fixed non-random limit distribution, then

(15) $\lim _{\substack{N, K \rightarrow \infty \\ \frac{K}{N}=\beta}} \mathbf{s}_{1}^{H}\left(\mathbf{S}_{1} \mathbf{D}_{1} \mathbf{S}_{1}^{H}\right)^{m} \mathbf{s}_{1} \stackrel{\text { a.s. }}{\rightarrow} \lim _{\substack{N, K \rightarrow \infty \\ \frac{K}{N}=\beta}} \frac{1}{N} \mathrm{E}\left[\operatorname{Trace}\left\{\left(\mathbf{S}_{1} \mathbf{D}_{1} \mathbf{S}_{1}^{H}\right)^{m}\right\}\right]=\int \lambda^{m} d G(\lambda)$,

where $G(\lambda)$ is the limit eigenvalue distribution of $\mathbf{S}_{1} \mathbf{D}_{1} \mathbf{S}_{1}^{H}$ and the convergence is almost surely. For proof see Appendix C.

Let $\mathbf{w}^{\infty}$ denote the asymptotic weight vector, from (15) it is readily shown that

$$
\mathbf{w}^{\infty}=\left[\begin{array}{ccc}
\mathcal{H}_{1}^{\infty}+A_{1}^{2} \mathcal{H}_{0}^{\infty} \mathcal{H}_{0}^{\infty} & \cdots & \mathcal{H}_{D}^{\infty}+A_{1}^{2} \mathcal{H}_{D-1}^{\infty} \mathcal{H}_{0}^{\infty} \\
\vdots & \ddots & \vdots \\
\mathcal{H}_{D}^{\infty}+A_{1}^{2} \mathcal{H}_{D-1}^{\infty} \mathcal{H}_{0}^{\infty} & \cdots & \mathcal{H}_{2 D-1}^{\infty}+A_{1}^{2} \mathcal{H}_{D-1}^{\infty} \mathcal{H}_{D-1}^{\infty}
\end{array}\right]^{-1}\left[\begin{array}{c}
\mathcal{H}_{0}^{\infty} \\
\vdots \\
\mathcal{H}_{D-1}^{\infty}
\end{array}\right]
$$

where 


$$
\mathcal{H}_{m}^{\infty}=\int\left(\lambda+\sigma^{2}\right)^{m} d G(\lambda)
$$

Now we can apply Proposition 1 to obtain $\mathcal{H}_{m}^{\infty}$ and therefore the asymptotic weights.

Proposition 1 requires that the empirical distribution of the received powers of the interfering users, which are the diagonal elements of $\mathbf{D}_{1}$, converges a.s. to a fixed non-random limit distribution. Based also on the observation given at beginning of the Section 2, we recall here that in order to verify the a.s. convergence of empirical distribution of the diagonal elements of $\mathbf{D}_{1}$ to a fixed non-random limit distribution it is enough to verify that all the moments of the matrix $\mathbf{D}_{1}$ exist, i.e. the limit in the following equation exists

$$
\lambda_{p}^{D} \triangleq \frac{1}{(K-1)} \lim _{K \rightarrow \infty} \mathrm{E}\left[\operatorname{Trace}\left\{\mathbf{D}_{1}^{p}\right\}\right]
$$

and that $\forall p$ the normalized trace of the matrix $\mathbf{D}_{1}^{p}$ converges a.s. to a non-random limit given by the moments of $\mathbf{D}_{1}$ i.e.:

$$
\frac{1}{(K-1)} \lim _{K \rightarrow \infty} \operatorname{Trace}\left\{\mathbf{D}_{1}^{p}\right\} \stackrel{a . s .}{\rightarrow} \lambda_{p}^{D} \quad 0 \leq p<\infty
$$

Notice also that the received power of each user is a positive random variable with a distribution function induced by the channel fading. If all the users experience independent fading, then a sufficient condition for the a.s. convergence of the empirical distribution of the interfering received powers to a non-random limit is given in $[3$, Theorem 2.3] in terms of the diagonal elements of the matrices $\mathbf{D}_{1}^{p}$ with $0 \leq p<\infty$. It easy to verify that most cases of practical interest satisfy those conditions. In particular, assuming that all the users experience i.i.d fading with uniformly bounded transmitted powers, it can be easily shown that if the common distribution of the users' fading coefficients is $p$-integrable with $0 \leq p<\infty$, then the the empirical distribution of the received powers converges a.s. to a non-random limit. Certainly, in all cases, this limit depends on the fading coefficients distribution and, consequently, it is subject to change if the channel characteristics change considerably. In practice, this means we need to update the distribution of the received powers periodically or when we detect a considerable change in it. In the implementation of the asymptotic reduced-rank receiver, we can use the moments of the empirical distribution of $A_{2}^{2}, \ldots, A_{K}^{2}$ when $K$ is finite to approximate the moments of the limit empirical distribution of the received powers used in (1), i.e.

$$
\mathrm{E}\left[\Lambda^{m}\right] \approx \frac{1}{K-1} \sum_{k=2}^{K} A_{k}^{2 m} .
$$

(18) is in fact what we use in the numerical results of this paper. 
3.1.2. Reduced-Rank Multiantenna MMSE Receiver. In a multiantenna DS-CDMA system, the output of the chip-matched filter at the $l$ th antenna element is

$$
\mathbf{r}_{l}=\sum_{k=1}^{K} A_{k} \mathrm{e}^{j \phi_{k}(l)} b_{k} \mathbf{s}_{k}+\mathbf{n}_{l} \quad l=1, \ldots, L .
$$

In the equation above, we assume that for each user, the received amplitudes at different antenna elements are the same for simplification. This is valid if the antenna elements are close enough to each other so that for a given user, the received powers at different antenna elements vary little. However, the received phases still vary considerably among antenna elements because different propagation paths have a much greater impact on the received phases due to the usually high carrier frequency.

Put in matrix form

$$
\overline{\mathbf{r}}=\overline{\mathbf{S}} \mathbf{A} \mathbf{b}+\overline{\mathbf{n}}
$$

where $\overline{\mathbf{r}}=\left(\mathbf{r}_{1}^{T}, \ldots, \mathbf{r}_{L}^{T}\right)^{T}$ is an $L N$ - vector, $\overline{\mathbf{S}}=\left(\overline{\mathbf{s}}_{1}, \ldots, \overline{\mathbf{s}}_{K}\right)$ is an $(L N) \times K$ matrix, $\overline{\mathbf{s}}_{k}=\left(\mathbf{s}_{k}^{T} \mathrm{e}^{j \phi_{k}(1)}, \ldots, \mathbf{s}_{k}^{T} \mathrm{e}^{j \phi_{k}(L)}\right)^{T}, \mathbf{A}=\operatorname{diag}\left\{A_{1}, \ldots, A_{K}\right\}, \mathbf{b}=\left(b_{1}, \ldots, b_{K}\right)^{T}$, and $\overline{\mathbf{n}}=\left(\mathbf{n}_{1}^{T}, \ldots, \mathbf{n}_{L}^{T}\right)^{T}$. The MMSE receiver for user 1 is

$$
\mathbf{c}=\left(\overline{\mathbf{S}}_{1} \mathbf{D}_{1} \overline{\mathbf{S}}_{1}^{H}+\sigma^{2} \mathbf{I}\right)^{-1} \overline{\mathbf{s}}_{1}
$$

where $\overline{\mathbf{S}}_{1}=\left(\overline{\mathbf{s}}_{2}, \ldots, \overline{\mathbf{s}}_{K}\right)$, and $\mathbf{D}_{1}=\operatorname{diag}\left\{A_{2}^{2}, \ldots, A_{K}^{2}\right\}$. The $\operatorname{rank} D(D \leq L N)$ reduced-rank MMSE receiver for user 1 is

$$
\mathbf{c}_{D}=\sum_{m=0}^{D-1} w_{m}\left(\overline{\mathbf{S}}_{1} \mathbf{D}_{1} \overline{\mathbf{S}}_{1}^{H}+\sigma^{2} \mathbf{I}\right)^{m} \overline{\mathbf{s}}_{1}
$$

where the weight vector $\mathbf{w}=\left(w_{0}, \ldots, w_{D-1}\right)^{T}$ is given by $(13)$, and

$$
\mathcal{H}_{m}=\overline{\mathbf{s}}_{1}^{H}\left(\overline{\mathbf{S}}_{1} \mathbf{D}_{1} \overline{\mathbf{S}}_{1}^{H}+\sigma^{2} \mathbf{I}\right)^{m} \overline{\mathbf{s}}_{1} .
$$

To calculate the limit of $\mathcal{H}_{m}$ as $N$ and $K$ go to infinity, we notice that the situation here is similar to the situation in 3.1.1, the only difference being that the energy (defined by $\mathrm{E}\left\{\|\cdot\|^{2}\right\}$ ) of $\overline{\mathbf{s}}_{1}$ and every column of $\overline{\mathbf{S}}_{1}$ is now $L$. After taking out the $L$ factor from $\overline{\mathbf{s}}_{1}$ and absorbing the $L$ factor of $\overline{\mathbf{S}}_{1}$ into $\mathbf{D}_{1}$, we have that

$$
\mathcal{H}_{m}^{\infty}=L \int\left(\lambda+\sigma^{2}\right)^{m} d G(\lambda)
$$

where $G(\lambda)$ is the limit eigenvalue distribution of $\tilde{\mathbf{S}}_{1}\left(L \mathbf{D}_{1}\right) \tilde{\mathbf{S}}_{1}^{H}$ with $\tilde{\mathbf{S}}_{1}=\overline{\mathbf{S}}_{1} / \sqrt{L}$. Proposition 2 can be applied to find $\mathcal{H}_{m}^{\infty}$ and therefore the asymptotic weights (16). 
3.1.3. Reduced-Rank Single Antenna LCM Receiver. The single antenna LCM receiver for user 1 is [16]

$$
\mathbf{c}=\mathbf{F}\left(\mathbf{S}_{1 a} \mathbf{D}_{1} \mathbf{S}_{1 a}^{H}+\sigma^{2} \mathbf{I}\right)^{-1} \mathbf{s}_{1 a},
$$

where $\mathbf{F}=\left[\begin{array}{ll}\mathbf{I}_{N} & \mathbf{0}\end{array}\right], \mathbf{I}_{N}$ is an $N \times N$ identity matrix, and

$$
\mathbf{s}_{1 a}=\left[\begin{array}{c}
\mathbf{s}_{1} \mathrm{e}^{j \phi_{1}} \\
\mathbf{s}_{1} \mathrm{e}^{-j \phi_{1}}
\end{array}\right], \mathbf{S}_{1 a}=\left[\begin{array}{ccc}
\mathbf{s}_{2} \mathrm{e}^{j \phi_{2}} & \cdots & \mathbf{s}_{K} \mathrm{e}^{j \phi_{K}} \\
\mathbf{s}_{2} \mathrm{e}^{-j \phi_{2}} & \cdots & \mathbf{s}_{K} \mathrm{e}^{-j \phi_{K}}
\end{array}\right] .
$$

The rank $D(D \leq 2 N)$ reduced-rank LCM receiver for user 1 is [16]

$$
\mathbf{c}_{D}=\sum_{m=0}^{D-1} w_{m}\left(\mathbf{S}_{1 a} \mathbf{D}_{1} \mathbf{S}_{1 a}^{H}+\sigma^{2} \mathbf{I}\right)^{m} \mathbf{s}_{1 a}
$$

with the weight vector $\mathbf{w}=\left(w_{0}, \ldots, w_{D-1}\right)^{T}$ given by [16]

$$
\mathbf{w}=\left[\begin{array}{ccc}
\mathcal{H}_{1} & \cdots & \mathcal{H}_{D} \\
\vdots & \ddots & \vdots \\
\mathcal{H}_{D} & \cdots & \mathcal{H}_{2 D-1}
\end{array}\right]^{-1}\left[\begin{array}{c}
\mathcal{H}_{1} \\
\vdots \\
\mathcal{H}_{D}
\end{array}\right]
$$

where

$$
\mathcal{H}_{m}=\mathbf{s}_{1 a}^{H}\left(\mathbf{S}_{1 a} \mathbf{D}_{1} \mathbf{S}_{1 a}^{H}+\sigma^{2} \mathbf{I}\right)^{m} \mathbf{s}_{1 a} .
$$

After taking out the factor of 2 from $\mathbf{s}_{1 a}$ and $\mathbf{S}_{1 a}$

$$
\mathcal{H}_{m}^{\infty}=2^{m+1} \int\left(\lambda+\frac{\sigma^{2}}{2}\right)^{m} d G(\lambda)
$$

where $G(\lambda)$ is the asymptotic eigenvalue distribution of $\tilde{\mathbf{S}}_{1 a} \mathbf{D}_{1} \tilde{\mathbf{S}}_{1 a}^{H}$ with $\tilde{\mathbf{S}}_{1 a}=\mathbf{S}_{1 a} / \sqrt{2}$. Proposition 3 can be applied to find $\mathcal{H}_{m}^{\infty}$ and the asymptotic weight vector $\mathbf{w}^{\infty}$.

3.1.4. Reduced-Rank Multiantenna LCM Receiver. As discussed in 3.1.2, the output of the chip-matched filter in a multiantenna system is

$$
\overline{\mathbf{r}}=\overline{\mathbf{S}} \mathbf{A b}+\overline{\mathbf{n}} .
$$

Similar to the single antenna case, the multiantenna LCM receiver for user 1 is

$$
\mathbf{c}=\mathbf{F}\left(\overline{\mathbf{S}}_{1 a} \mathbf{D}_{1} \overline{\mathbf{S}}_{1 a}^{H}+\sigma^{2} \mathbf{I}\right)^{-1} \overline{\mathbf{s}}_{1 a}
$$


where $\mathbf{F}=\left[\begin{array}{ll}\mathbf{I}_{L N} & \mathbf{0}\end{array}\right]$, and

$$
\overline{\mathbf{s}}_{1 a}=\left[\begin{array}{c}
\overline{\mathbf{s}}_{1} \\
\overline{\mathbf{s}}_{1}^{*}
\end{array}\right], \overline{\mathbf{S}}_{1 a}=\left[\begin{array}{ccc}
\overline{\mathbf{s}}_{2} & \cdots & \overline{\mathbf{s}}_{K} \\
\overline{\mathbf{s}}_{2}^{*} & \cdots & \overline{\mathbf{s}}_{K}^{*}
\end{array}\right] .
$$

The rank $D(D \leq 2 L N)$ reduced-rank LCM receiver for user 1 is

$$
\mathbf{c}_{D}=\sum_{m=0}^{D-1} w_{m}\left(\overline{\mathbf{S}}_{1 a} \mathbf{D}_{1} \overline{\mathbf{S}}_{1 a}^{H}+\sigma^{2} \mathbf{I}\right)^{m} \overline{\mathbf{s}}_{1 a}
$$

where the weight vector $\mathbf{w}=\left(w_{0}, \ldots, w_{D-1}\right)^{T}$ is given by $(28)$, and

$$
\mathcal{H}_{m}=\overline{\mathbf{s}}_{1 a}^{H}\left(\overline{\mathbf{S}}_{1 a} \mathbf{D}_{1} \overline{\mathbf{S}}_{1 a}^{H}+\sigma^{2} \mathbf{I}\right)^{m} \overline{\mathbf{s}}_{1 a} .
$$

To calculate the limit of $\mathcal{H}_{m}$ as $N$ and $K$ go to infinity, we notice that the energy (defined by $\mathrm{E}\left\{\|\cdot\|^{2}\right\}$ ) of $\overline{\mathbf{s}}_{1 a}$ and every column of $\overline{\mathbf{S}}_{1 a}$ is $2 L$. By following similar arguments in Section (3.1.2) and (3.1.3), it is easily shown that

$$
\mathcal{H}_{m}^{\infty}=L 2^{m+1} \int\left(\lambda+\frac{\sigma^{2}}{2}\right)^{m} d G(\lambda)
$$

where $G(\lambda)$ is the asymptotic eigenvalue distribution of $\tilde{\mathbf{S}}_{1 a}\left(L \mathbf{D}_{1}\right) \tilde{\overline{\mathbf{S}}}_{1 a}^{H}$ with $\tilde{\mathbf{S}}_{1 a}=$ $\overline{\mathbf{S}}_{1 a} / \sqrt{2 L}$. Proposition 4 can be applied to find $\mathcal{H}_{m}^{\infty}$ and the asymptotic weight vector $\mathbf{w}^{\infty}$.

3.1.5. Reduced-Rank Multiantenna MMSE Receiver in Line-of-Sight Transmission. In a line-of-sight transmission DS-CDMA system with multiple receive antenna, the output of the chip-matched filter at the $l$ th antenna element is

$$
\mathbf{r}_{l}=\sum_{k=1}^{K} A_{k} \mathrm{e}^{l j \phi_{k}} b_{k} \mathbf{s}_{k}+\mathbf{n}_{l} \quad l=0, \ldots, L-1,
$$

where $\phi_{k}=2 \pi d \cos \alpha_{k} / \lambda, d$ is the distance between two adjacent antenna elements, $\alpha_{k}$ is the incident angle of user $k$, and $\lambda$ is the wavelength of the carrier. Due to the randomness in the geographic distribution of users, it is reasonable to assume that $\phi_{k}$ 's are i.i.d. random variables uniformly distributed on $[0,2 \pi)$. Put in matrix form

$$
\overline{\mathbf{r}}=\overline{\mathbf{S}} \mathbf{A b}+\overline{\mathbf{n}},
$$

where $\overline{\mathbf{r}}=\left(\mathbf{r}_{0}^{T}, \ldots, \mathbf{r}_{L-1}^{T}\right)^{T}$ is an $L N$-vector, $\overline{\mathbf{S}}=\left(\overline{\mathbf{s}}_{1}, \ldots, \overline{\mathbf{s}}_{K}\right)$ is an $(L N) \times K$ matrix, $\overline{\mathbf{s}}_{k}=\left(\mathbf{s}_{k}^{T} \mathrm{e}^{0 j \phi_{k}}, \ldots, \mathbf{s}_{k}^{T} \mathrm{e}^{(L-1) j \phi_{k}}\right)^{T}, \mathbf{A}=\operatorname{diag}\left\{A_{1}, \ldots, A_{K}\right\}, \mathbf{b}=\left(b_{1}, \ldots, b_{K}\right)^{T}$, and $\overline{\mathbf{n}}=\left(\mathbf{n}_{1}^{T}, \ldots, \mathbf{n}_{L}^{T}\right)^{T}$. The MMSE receiver for user 1 is 


$$
\mathbf{c}=\left(\overline{\mathbf{S}}_{1} \mathbf{D}_{1} \overline{\mathbf{S}}_{1}^{H}+\sigma^{2} \mathbf{I}\right)^{-1} \overline{\mathbf{s}}_{1},
$$

where $\overline{\mathbf{S}}_{1}=\left(\overline{\mathbf{s}}_{2}, \ldots, \overline{\mathbf{s}}_{K}\right)$, and $\mathbf{D}_{1}=\operatorname{diag}\left\{A_{2}^{2}, \ldots, A_{K}^{2}\right\}$. The rank $D(D \leq L N)$ reduced-rank MMSE receiver for user 1 is

$$
\mathbf{c}_{D}=\sum_{m=0}^{D-1} w_{m}\left(\overline{\mathbf{S}}_{1} \mathbf{D}_{1} \overline{\mathbf{S}}_{1}^{H}+\sigma^{2} \mathbf{I}\right)^{m} \overline{\mathbf{s}}_{1}
$$

with the weights given by (13) and

$$
\mathcal{H}_{m}=\overline{\mathbf{s}}_{1}^{H}\left(\overline{\mathbf{S}}_{1} \mathbf{D}_{1} \overline{\mathbf{S}}_{1}^{H}+\sigma^{2} \mathbf{I}\right)^{m} \overline{\mathbf{s}}_{1} .
$$

The asymptotic weights can be obtained by using Proposition 5 in the same way we used Proposition 2 in 3.1.2.

\subsubsection{Reduced-Rank Multiantenna LCM Receiver in Line-of-Sight}

Transmission. As discussed in 3.1.5, the output of the chip-matched filter in a lineof-sight transmission DS-CDMA system is

$$
\overline{\mathbf{r}}=\overline{\mathbf{S}} \mathbf{A b}+\overline{\mathbf{n}}
$$

Similar to the discussion in 3.1.4, the LCM receiver for user 1 is

$$
\mathbf{c}=\mathbf{F}\left(\overline{\mathbf{S}}_{1 a} \mathbf{D}_{1} \overline{\mathbf{S}}_{1 a}^{H}+\sigma^{2} \mathbf{I}\right)^{-1} \overline{\mathbf{s}}_{1 a},
$$

where $\mathbf{F}=\left[\begin{array}{ll}\mathbf{I}_{L N} & \mathbf{0}\end{array}\right]$, and

$$
\overline{\mathbf{s}}_{1 a}=\left[\begin{array}{c}
\overline{\mathbf{s}}_{1} \\
\overline{\mathbf{s}}_{1}^{*}
\end{array}\right], \overline{\mathbf{S}}_{1 a}=\left[\begin{array}{lll}
\overline{\mathbf{s}}_{2} & \cdots & \overline{\mathbf{s}}_{K} \\
\overline{\mathbf{s}}_{2}^{*} & \cdots & \overline{\mathbf{s}}_{K}^{*}
\end{array}\right] .
$$

The rank $D(D \leq 2 L N)$ reduced-rank LCM receiver for user 1 is

$$
\mathbf{c}_{D}=\sum_{m=0}^{D-1} w_{m}\left(\overline{\mathbf{S}}_{1 a} \mathbf{D}_{1} \overline{\mathbf{S}}_{1 a}^{H}+\sigma^{2} \mathbf{I}\right)^{m} \overline{\mathbf{s}}_{1 a}
$$

with the weights given by (28) and

$$
\mathcal{H}_{m}=\overline{\mathbf{s}}_{1 a}^{H}\left(\overline{\mathbf{S}}_{1 a} \mathbf{D}_{1} \overline{\mathbf{S}}_{1 a}^{H}+\sigma^{2} \mathbf{I}\right)^{m} \overline{\mathbf{s}}_{1 a} .
$$

The asymptotic weights can be obtained by using Proposition 6 in the same way we used Proposition 4 in 3.1.4. 
3.2. Relationships among Asymptotic Eigenvalue Distributions in Various Cases. In Section 2, the moments of the asymptotic eigenvalue distributions of several classes of random matrices are shown to be equal. In this section, we study the relationships among these distributions through the moments. And because the asymptotic output SINR is a certain integrable function averaged with respect to the asymptotic eigenvalue distribution, the relationships among them are also characterized.

The multiantenna LCM receiver serves as an example to illustrate the idea. Similar results on the multiantenna MMSE receiver and the single antenna LCM receiver were obtained in [8] and [16], respectively, by using free probability theory.

Similar to the result on the single antenna receiver in [16], the output SINR of the multiantenna $\mathrm{LCM}$ receiver is

$$
P_{1} \overline{\mathbf{s}}_{1 a}^{H}\left(\overline{\mathbf{S}}_{1 a} \mathbf{D}_{1} \overline{\mathbf{S}}_{1 a}^{H}+\sigma^{2} \mathbf{I}\right)^{-1} \overline{\mathbf{s}}_{1 a}
$$

where $\mathbf{D}_{1}=\operatorname{diag}\left\{P_{2}, \ldots, P_{K}\right\}$, and $P_{k}=A_{k}^{2}$ is the received power of user $k$ at one antenna element. $\overline{\mathbf{s}}_{1 a}$ and $\overline{\mathbf{S}}_{1 a}$ are given by (33). We notice that in (47), the energy of $\overline{\mathbf{s}}_{1 a}$ and each column of $\overline{\mathbf{S}}_{1 a}$ (defined as $\mathrm{E}\left\{\|\cdot\|^{2}\right\}$ ) is $2 L$. After absorbing the factor $L$ of $\overline{\mathbf{s}}_{1 a}$ and $\overline{\mathbf{S}}_{1 a}$ into $P_{1}$ and $\mathbf{D}_{1}$, and taking the factor 2 of $\overline{\mathbf{S}}_{1 a}$ out of the matrix inversion, we have

$$
P_{1} \overline{\mathbf{s}}_{1 a}^{H}\left(\overline{\mathbf{S}}_{1 a} \mathbf{D}_{1} \overline{\mathbf{S}}_{1 a}^{H}+\sigma^{2} \mathbf{I}\right)^{-1} \overline{\mathbf{s}}_{1 a}=\left(L P_{1}\right) \tilde{\overline{\mathbf{s}}}_{1 a}^{H}\left(\tilde{\overline{\mathbf{S}}}_{1 a}\left(L \mathbf{D}_{1}\right) \tilde{\overline{\mathbf{S}}}_{1 a}^{H}+\frac{\sigma^{2}}{2} \mathbf{I}\right)^{-1} \tilde{\overline{\mathbf{s}}}_{1 a}
$$

where tilded quantities denote the normalized untilded ones. Now we consider a single antenna MMSE receiver in a DS-CDMA system with processing gain $2 L N$, number of users $K$, noise level $\sigma^{2} / 2$, and received power of user $k L P_{k}$, its output SINR is

$$
\left(L P_{1}\right) \mathbf{s}_{1}^{H}\left(\mathbf{S}_{1}\left(L \mathbf{D}_{1}\right) \mathbf{S}_{1}^{H}+\frac{\sigma^{2}}{2} \mathbf{I}\right)^{-1} \mathbf{s}_{1},
$$

where the $2 L N$-vector $\mathbf{s}_{k}$ is the spreading sequence of user $k, \mathbf{S}_{1}=\left(\mathbf{s}_{2}, \ldots, \mathbf{s}_{K}\right)$, $\mathbf{D}_{1}=\operatorname{diag}\left\{P_{2}, \ldots, P_{K}\right\}$, and $P_{k}=A_{k}^{2}$. All the spreading sequences are normalized. Suppose the asymptotic eigenvalue distribution of $\mathbf{S}_{1}\left(L \mathbf{D}_{1}\right) \mathbf{S}_{1}^{H}$ is $G(\lambda)$, the asymptotic eigenvalue distribution of $\tilde{\overline{\mathbf{S}}}_{1 a}\left(L \mathbf{D}_{1}\right) \tilde{\overline{\mathbf{S}}}_{1 a}^{H}$ is $G^{*}(\lambda)$, then from Proposition 4

$$
\int \lambda^{m} d G(\lambda)=\int \lambda^{m} d G^{*}(\lambda) \quad m=0,1,2, \ldots
$$

Denote the distribution density functions of $G$ and $G^{*}$ by $g$ and $g^{*}$, we have

$$
\int \lambda^{m}\left(g(\lambda)-g^{*}(\lambda)\right) d \lambda=0 \quad m=0,1,2, \ldots
$$


So, $g-g^{*}$ is orthogonal to every polynomial, therefore $g-g^{*}$ is zero almost everywhere, as a result $G=G^{*}$ almost everywhere. From [15]

$$
\mathbf{s}_{1}^{H}\left(\mathbf{S}_{1}\left(L \mathbf{D}_{1}\right) \mathbf{S}_{1}^{H}+\frac{\sigma^{2}}{2} \mathbf{I}\right)^{-1} \mathbf{s}_{1} \rightarrow \int \frac{1}{\lambda+\frac{\sigma^{2}}{2}} d G(\lambda)
$$

and from a similar argument in the proof of Lemma 4.3 in [15]

$$
\tilde{\mathbf{\mathbf { S }}}_{1 a}^{H}\left(\tilde{\overline{\mathbf{S}}}_{1 a}\left(L \mathbf{D}_{1}\right) \tilde{\overline{\mathbf{S}}}_{1 a}^{H}+\frac{\sigma^{2}}{2} \mathbf{I}\right)^{-1} \tilde{\mathbf{\mathbf { s }}}_{1 a} \rightarrow \int \frac{1}{\lambda+\frac{\sigma^{2}}{2}} d G^{*}(\lambda) .
$$

Therefore the asymptotic output SINR of the single antenna MMSE receiver with $\left(K, 2 L N, \sigma^{2} / 2\right)$ is the same as that of the multiantenna $\mathrm{LCM}$ receiver with $\left(K, N, \sigma^{2}\right)$. Similar results hold for the MMSE/LCM receivers in line-of-sight transmission.

REMARK. In [8], the output SINR of the multiantenna MMSE receiver with $\left(K, N, \sigma^{2}\right)$ and $L$ antenna elements is shown to be equal to that of the single antenna MMSE receiver with $\left(K, L N, \sigma^{2}\right)$ asymptotically. In that proof, free probability theory was used to obtain the asymptotic equality. By using the combinatorial techniques provided in the appendices of this paper, it can be shown that the expected values (with respect to the spreading sequences) of the SINR's of both systems are equal even for $K$ and $N$ finite. For the LCM receiver, however, since there are conjugate parts in the effective spreading sequences, from the proof in Appendix B, we need to let $K$ and $N$ go to infinity to make some terms vanish. Therefore, it is still open whether the expected values (with respect to the spreading sequences) of the SINR's of both systems (single antenna MMSE with $\left(K, 2 L N, \sigma^{2} / 2\right)$ and multiantenna LCM with $\left.\left(K, N, \sigma^{2}\right)\right)$ are equal for $K$ and $N$ finite. Since the output SINR's are random variables for $K$ and $N$ finite, they themselves are generally not equal when $K$ and $N$ are finite.

4. Deriving the Tse-Hanly Formula by Means of the Moments. By using the Silverstein-Bai theorem [13], it is shown in [15] that the asymptotic output SINR $\gamma$ of the MMSE receiver in a DS-CDMA system with random spreading satisfies the following fixed point equation (without loss of generality, the desired user is received with unit power)

$$
\gamma=\frac{1}{\sigma^{2}+\int \frac{\beta P}{1+P \gamma} d F(P)}
$$

where $F(P)$ is the limit of the empirical distribution of $P_{2}, \ldots, P_{K}$ as $K$ goes to infinity. In this section, we give a self-contained proof of (54) using the combinatorial convolution defined on the incidence algebra of non-crossing partitions. Based on these tools, we reduce (54) to a combinatorial equality involving the asymptotic moments. 
Let $G(\lambda)$ be the asymptotic eigenvalue distribution of $\mathbf{S}_{1} \mathbf{D}_{1} \mathbf{S}_{1}^{H}$. If $G(\lambda)$ is a compactly supported probability measure on $\mathbb{R}$, its Stieltjes transform is defined as:

$$
m(z)=\int \frac{1}{\lambda-z} d G(\lambda)
$$

which is an analytic function in $\mathcal{C} \backslash \operatorname{supp}(G)$. By expanding $\frac{1}{\lambda-z}$ with respect to $z$, exchanging the summation and integration and by analytical extension, (55) can be written $\forall z \in \mathcal{C} \backslash \operatorname{supp}(G)$ as:

$$
m(z)=-\frac{1}{z} \sum_{k=0}^{\infty} \frac{\lambda_{k}}{z^{k}}
$$

where

$$
\lambda_{k}=\int \lambda^{k} d G(\lambda)
$$

is given in Proposition 1. Notice that regardless of $G(\lambda), \mathcal{C} \backslash \operatorname{supp}(G) \supset z \in \mathcal{C}^{+}=\{z \in$ $\mathcal{C}: \operatorname{Im}(z)>0\}$. Notice that if $z \in \mathcal{C}^{+}$then also $m(z) \in \mathcal{C}^{+}$. To avoid cumbersome negative powers of -1 we denote $g(z)=-m(z)$, so

$$
g(z)=\frac{1}{z}+\sum_{k=1}^{\infty} \frac{\lambda_{k}}{z^{k+1}}=\sum_{k=1}^{\infty} \frac{\lambda_{k-1}}{z^{k}} .
$$

As in Remark 3.3.3 in [19], $g(z)$ has a unique inverse $k(\zeta)$, i.e. $\zeta=g(z), z=k(\zeta)$,

$$
k(\zeta)=\frac{1}{\zeta}+\sum_{k=0}^{\infty} a_{k+1} \zeta^{k},
$$

where $a_{k}$ is a polynomial in $\lambda_{1}, \ldots, \lambda_{k}$. Thus, from (59) we have:

$$
z=k(g(z))=\frac{1}{g(z)}+\sum_{k=0}^{\infty} a_{k+1} g(z)^{k},
$$

or equivalently:

$$
z g(z)=\sum_{k=1}^{\infty} \frac{\lambda_{k}}{z^{k}}=\sum_{k=0}^{\infty} a_{k+1} g(z)^{k+1} .
$$

Finally, from (61) we get the following equality between two power series:

$$
\sum_{k=1}^{\infty} \lambda_{k} x^{k}=\sum_{k=1}^{\infty} a_{k} y^{k},
$$

with

$$
x=\frac{1}{z}, \quad y=\sum_{k=1}^{\infty} \lambda_{k-1} x^{k} .
$$


Eq. (62) is satisfied when the sequence $\left(a_{1}, a_{2}, \ldots\right)$ satisfies the following implicit equation [6]:

$$
\lambda_{m}=\sum_{k=1}^{m} a_{k} \sum_{m_{1}+\cdots+m_{k}=m} \lambda_{m_{1}-1} \cdots \lambda_{m_{k}-1} .
$$

The series $\sum_{k=0}^{\infty} a_{k} \zeta^{k}$ is known as the R-transform of the asymptotic eigenvalue distribution, $G(\lambda)$, and the series coefficients $\left\{a_{k}\right\}$ are known as the free cumulants of $G(\lambda)$ $[19,14]$. In the following we will prove an explicitly expression for the free cumulants $\left\{a_{k}\right\}$ :

$$
a_{k}=\mathrm{E}\left\{\Lambda^{k}\right\} \beta,
$$

where $\Lambda$ is a random variable with cumulative distribution $F(P)$ as defined in Proposition 1 . The proof of (64) requires tools from set partition theory. Our treatment here is very brief; for more details please consult [1] and [14].

Definition 1. Let $m$ be a natural number. A partition $\pi \triangleq\left\{V_{1}, \ldots, V_{k}\right\}$ of the set $\{1, \ldots, m\}$ is a decomposition of $\{1, \ldots, m\}$ into disjoint and non-empty sets $V_{i}$ such that $\bigcup_{i=1}^{k} V_{i}=\{1, \ldots, m\}$. The elements $V_{i}$ are called the blocks of the partition $\pi$. We will denote the set of all partitions of $\{1, \ldots, m\}$ by $\mathcal{P}(m)$. This set becomes a lattice if we introduce the following partial order (called refinement order): $\pi \leq \sigma$ if each block of $\sigma$ is a union of blocks of $\pi$. We will denote the smallest and the biggest elements of $\mathcal{P}(m)$ - consisting of $m$ blocks and one block, by:

$$
0_{m}=\{(1),(2), \ldots,(m)\}, \quad 1_{m}=\{(1,2, \ldots, m)\}
$$

Definition 2. A partition $\pi \in \mathcal{P}(m)$ is a crossing partition if there exist four numbers $1 \leq i<k<j<l<m$ such that $i$ and $j$ are in the same block $V, k$ and $l$ are in the same block $V^{\prime}$ and $V \neq V^{\prime}$. The set of all non-crossing partitions in $\mathcal{P}(m)$ is denoted by $\mathrm{NC}(m)$, i.e:

$$
\mathrm{NC}(m) \triangleq\{\pi \in \mathcal{P}(m) \mid \pi \text { non - crossing }\}
$$

Define interval in $\mathrm{NC}(m)$ as the following set $[\pi, \sigma]=\{\tau \in \mathrm{NC}(m) \mid \pi \leq \tau \leq \sigma\}$ $\forall \pi \leq \sigma \in \mathrm{NC}(m)$. Each of these intervals can be decomposed into a product of lattices of non-crossing partitions, i.e. for all $\pi, \sigma \in \mathrm{NC}(m)$ with $\pi \leq \sigma$ there exist canonical natural numbers $k_{1}, k_{2}, \ldots$, such that

$$
[\pi, \sigma] \cong \mathrm{NC}(1)^{k_{1}} \times \mathrm{NC}(2)^{k_{2}} \times \cdots
$$

For example we have:

$$
\begin{gathered}
{[\{(1),(2),(3),(4),(5),(6),(7),(8)\},\{(1,2,3),(4,5),(6),(7,8)\}]_{\mathrm{NC}(8)} \cong} \\
\cong[\{(1),(2),(3)\},\{(1,2,3)\}]_{\mathrm{NC}(3)} \times[\{(4),(5)\},\{(4,5)\}]_{\mathrm{NC}(2)} \\
\times[(6),(6)]_{\mathrm{NC}(1)} \times[\{(7),(8)\},\{(7,8)\}]_{\mathrm{NC}(2)} \cong \\
\cong \mathrm{NC}(1) \times \mathrm{NC}(2)^{2} \times \mathrm{NC}(3)
\end{gathered}
$$


Having this factorization property at hand it is quite natural to define a multiplicative function $f$ (for non-crossing partitions) corresponding to a sequence $\left(a_{1}, a_{2}, \ldots\right)$ of complex numbers by requirement that:

$$
f(\pi, \sigma) \triangleq\left\{\begin{array}{c}
a_{1}^{k_{1}} a_{2}^{k_{2}} \cdots \\
0
\end{array} \quad \text { whenever } \pi \not \leq \sigma\right.
$$

if $[\pi, \sigma]$ has a factorization as above. We use the notation $f \leadsto\left(a_{1}, a_{2}, \ldots\right)$ to denote the dependence of $f$ on the sequence $\left(a_{1}, a_{2}, \ldots\right)$. In particular we have that $f\left(0_{m}, 1_{m}\right)=a_{m}$

Definition 3. The (combinatorial) convolution $(\cdot * \cdot)$ of $f$ and $g$ defined as in $(68),(f * g)$, is equal to:

$$
\begin{aligned}
(f * g) \triangleq \sum_{\substack{\tau \in \mathrm{NC}(m) \\
\pi \leq \tau \leq \sigma}} f(\pi, \tau) g(\tau, \sigma) \quad \text { for } \pi \leq \sigma \in \mathrm{NC}(m) . \\
\end{aligned}
$$

Now we have the machinery to prove (54). According to Proposition 1:

$$
\begin{aligned}
\lambda_{m} & =\sum_{k=1}^{m} \beta^{k} \sum_{m_{1}+\cdots+m_{k}=m} c\left(m_{1}, \ldots, m_{k}\right) \cdot \mathrm{E}\left\{\Lambda^{m_{1}}\right\} \cdots \mathrm{E}\left\{\Lambda^{m_{k}}\right\} \\
& =\sum_{\substack{m_{1}+\cdots+m_{k}=m \\
1 \leq k \leq m}} c\left(m_{1}, \ldots, m_{k}\right) \cdot Q_{m_{1}} \cdots Q_{m_{k}},
\end{aligned}
$$

where $Q_{m_{j}}=\mathrm{E}\left\{\Lambda^{m_{j}}\right\} \beta$. Define the following multiplicative functions on the lattice of non-crossing partitions:

- $L$ an $\left(\lambda_{1}, \lambda_{2}, \ldots\right)$ as the multiplicative function on the lattice of non-crossing partitions corresponding to the moments $\lambda_{m}$ of the random matrix $\mathbf{S D S}^{H}$,

- $Q \leadsto\left(Q_{1}, Q_{2}, \ldots\right)$ as the multiplicative function on the lattice of non-crossing partitions corresponding to the sequence $Q_{m}$.

It is easy to see that:

$$
\begin{aligned}
& \lambda_{m}=L\left(0_{m}, 1_{m}\right) \\
& Q(\pi, \sigma)=Q_{1}^{k_{1}} Q_{2}^{k_{2}} \cdots \quad \text { iff } \quad[\pi, \sigma] \cong \mathrm{NC}(1)^{k_{1}} \times \mathrm{NC}(2)^{k_{2}} \times \cdots
\end{aligned}
$$

From Theorem 2 in Appendix A, we know that $c\left(m_{1}, \ldots, m_{k}\right)$ is the number of noncrossing partitions of $m$ into $k$ disjoint sets, such that the numbers of elements in these sets are $m_{1}, \ldots, m_{k}$. As a consequence, from (69) and (71), (70) can be rewritten as

$$
\lambda_{m}=\sum_{\substack{\tau \in \mathrm{NC}(m) \\ 0_{m} \leq \tau \leq 1_{m}}} Q\left(0_{m}, \tau\right) \text { zet } a\left(\tau, 1_{m}\right)=\mathrm{Q} * \text { zeta }
$$


where zeta is the zeta function defined as in $[1,14]$ :

$$
z \operatorname{eta}(\pi, \sigma)= \begin{cases}1, & \pi \leq \sigma \\ 0, & \text { otherwise }\end{cases}
$$

By taking into account the non-crossing character of the involved partition, the relation $L=Q *$ zeta can be written more concretely in a recursive way as $\left(\lambda_{0}=1\right)$ [14]:

$$
\lambda_{m}=\sum_{k=1}^{m} Q_{k} \sum_{\substack{i_{1}, \ldots, i_{k} \geq 0 \\ i_{1}+\cdots+i_{k}=m}} \lambda_{i_{1}-1} \cdots \lambda_{i_{k}-1} .
$$

Comparing Eq. (63) with Eq. (74) it follows immediately that $a_{k}=Q_{k}=\mathrm{E}\left\{\Lambda^{k}\right\} \beta$, as we wanted to show. As a consequence,

$$
\begin{aligned}
k(g(z)) & =\frac{1}{g(z)}+\sum_{k=1}^{\infty} \mathrm{E}\left\{\Lambda^{k}\right\} \beta(g(z))^{k} \\
& =-\frac{1}{m(z)}+\sum_{k=1}^{\infty} \mathrm{E}\left\{\Lambda^{k}\right\} \beta(-1)^{k}(m(z))^{k} \\
& =-\frac{1}{m(z)}+\int \frac{\beta P}{1+\operatorname{Pm}(z)} d F(P) .
\end{aligned}
$$

For (75) we have used the fact that for $z \in \mathcal{C}^{+}$:

$$
\sum_{k=1}^{\infty} \mathrm{E}\left\{\Lambda^{k}\right\} \beta(-1)^{k} z^{k}=\int \frac{\beta P}{1+P z} d F(P)
$$

which can be easily shown by analytic extension expanding $\frac{1}{1+P z}$ with respect to $z$ and exchanging the summation and the integration with respect to $F(P)$. Since $k(g(z))=z$, so:

$$
m(z)=\frac{1}{-z+\int \frac{\beta P}{1+P m(z)} d F(P)}
$$

Therefore, via the asymptotic moments we obtain the same result originally shown by Silverstein and Bai [13].

Using Corollary 1 in [4] and the results in [21], it can be shown that if the empirical distribution of the diagonal elements of the matrix $\mathbf{D}_{1}$ converges almost surely (a.s.) to a fixed distribution, then as $N$ and $K$ go to infinity with $K / N=\beta$, the output SINR of the MMSE receiver for user $1, \gamma$, converges almost surely for $N \rightarrow \infty$ to :

$$
\gamma=\mathbf{s}_{1}^{H}\left(\mathbf{S}_{1} \mathbf{D}_{1} \mathbf{S}_{1}^{H}+\sigma^{2} \mathbf{I}\right)^{-1} \mathbf{s}_{1} \stackrel{a . s .}{\rightarrow} \lim _{N \rightarrow \infty} \frac{1}{N} \mathrm{E}\left[\operatorname{Trace}\left\{\left(\mathbf{S}_{1} \mathbf{D}_{1} \mathbf{S}_{1}^{H}+\sigma^{2} \mathbf{I}\right)^{-1}\right\}\right] .
$$


Since

(79)

$$
\lim _{N \rightarrow \infty} \frac{1}{N} \mathrm{E}\left[\operatorname{Trace}\left\{\left(\mathbf{S}_{1} \mathbf{D}_{1} \mathbf{S}_{1}^{H}+\sigma^{2} \mathbf{I}\right)^{-1}\right\}\right]=\lim _{z \rightarrow-\sigma^{2}} \int \frac{1}{\lambda-z} d G(\lambda)=\lim _{z \rightarrow-\sigma^{2}} m(z)
$$

letting $z=-\sigma^{2}$ in (77), (54) is obtained.

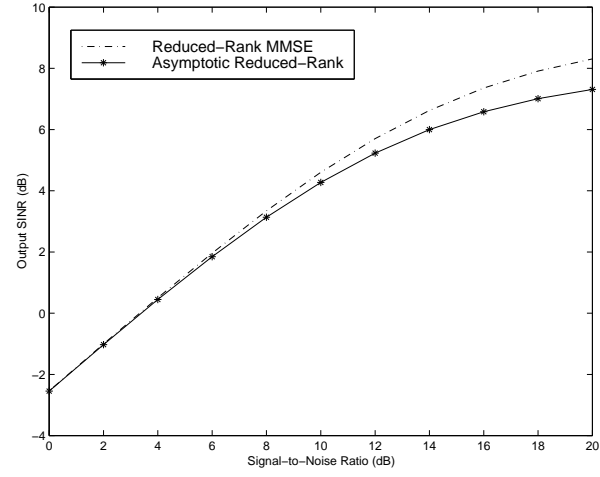

FIG. 1. Output SINR vs. input signal-tonoise ratio for two reduced-rank single antenna receivers.

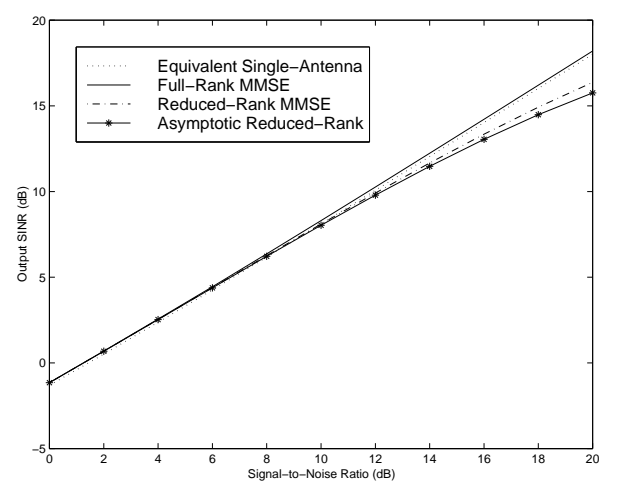

FIG. 2. Output SINR vs. input signal-tonoise ratio for multiantenna MMSE receivers.

5. Numerical Results. In this section, we compare the performance of four receivers: (i) the asymptotic reduced-rank MMSE/LCM receiver that uses the asymptotic weights; (ii) the reduced-rank MMSE/LCM receiver that uses the exact optimal weights; (iii) the full-rank MMSE/LCM receiver; (iv) the asymptotically equivalent single antenna MMSE receiver with enlarged processing gain and possibly reduced noise level. In all the results, $N=32, K=25, L=2$ (number of receive antenna elements), $D=5$ (number of stages of the reduced-rank receiver) are used. For simplification, we simulate the case in which the received powers of the interfering users are equal and are $5 \mathrm{~dB}$ above the desired user. This corresponds to power-controlled interferers and makes the desired user to operate in a relatively challenging environment. In the case of single antenna receivers, the horizontal axis represents the input signal-to-noise ratio $P_{1} / \sigma^{2}$ in $\mathrm{dB}$. For multiantenna receivers, the horizontal axis is $L P_{1} / \sigma^{2}$, taking into account the total received power of the desired user.

Figure 1 shows the performance of two reduced-rank single antenna MMSE receivers. The upper curve is the output SINR of the reduced-rank MMSE receiver that uses exact optimal weights, and the lower curve is for the asymptotic reduced-rank receiver that uses asymptotic weights. From the figure we can see that the later lies within $1 \mathrm{~dB}$ from the former. And we found that (not shown in the figure) if $N>80$, the performance loss due to using asymptotic weights will be less than $0.5 \mathrm{~dB}$.

Figure 2 shows the performance of the multiantenna MMSE receivers. From the figure we can see that we lose about $1 \mathrm{~dB}$ in SINR by using asymptotic weights. And as predicted, the performance of the full-rank MMSE receiver is very close to that 
of the asymptotically equivalent single antenna MMSE receiver with processing gain $L N$. We also found that (not shown in the figure), if $L N$ (the effective processing gain) is raised to above 100 , the penalty of using asymptotic weights will be less than $0.5 \mathrm{~dB}$, and if $D \geq 8$, the performance gap between the reduced-rank receiver and the full-rank one will be less than $0.5 \mathrm{~dB}$.

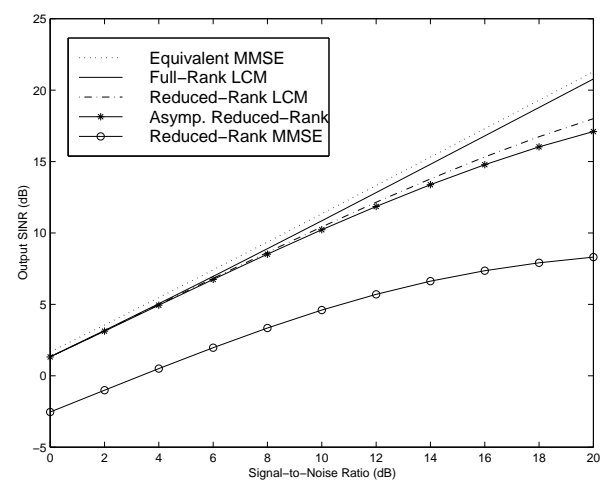

FIG. 3. Output SINR vs. input signal-tonoise ratio for single antenna $L C M$ receivers.

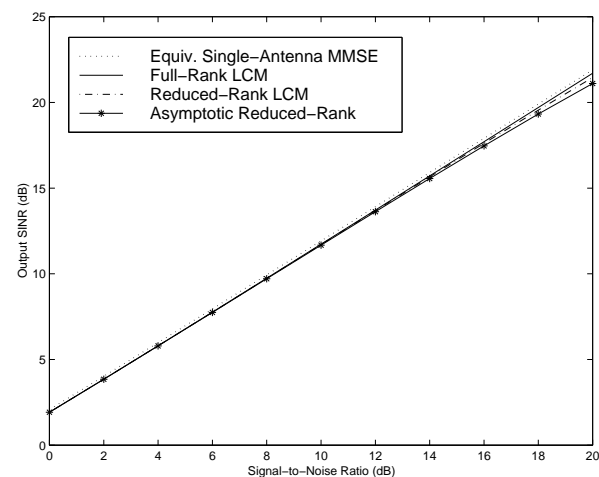

FIG. 4. Output SINR vs. input signal-tonoise ratio for multiantenna $L C M$ receivers.

Figure 3 illustrates the performance of the single antenna LCM receivers. We observe that the penalty due to using asymptotic weights is within $1.25 \mathrm{~dB}$. And as predicted, performance of the full-rank LCM receiver is very close to that of the asymptotically equivalent single antenna MMSE receiver with processing $2 N$ and noise level $\sigma^{2} / 2$. We found that (not shown in the figure) if $N>60$, the performance loss due to using asymptotic weights will be less than $0.5 \mathrm{~dB}$, and if $D \geq 8$, the performance gap between the reduced- rank receiver and the full-rank one will be less than $0.5 \mathrm{~dB}$. For comparison purposes, the output SINR of the reduced-rank MMSE receiver in Figure 1 is also shown here. We can see the significant performance gain obtained by LCM processing.

Figure 4 illustrates the performance of the multiantenna LCM receivers. From the figure we see that the performance loss due to using asymptotic weights is very small (about $0.5 \mathrm{~dB}$ ). Additionally the performance difference between the full-rank LCM receiver and the asymptotically equivalent single antenna MMSE receiver with processing $2 L N$ and with noise level $\sigma^{2} / 2$ is also very small, almost indistinguishable. This is because the effective processing $2 L N=128$, which is large enough to guarantee sufficient convergence. By comparison of Figures 2 and 4, we can see that the output SINR in Figure 4 is $4-5 \mathrm{~dB}$ higher than those in Figure 2. This confirms the analytical result given in (53) that the LCM receiver asymptotically reduces the number of interferers and the noise level by half.

Figure 5 illustrates the performance of the MMSE receivers in line-of- sight transmission. We see that Figure 5 quite resembles Figure 2. This agrees with our analytical result that their performances are asymptotically equal. All observations in Figure 2 


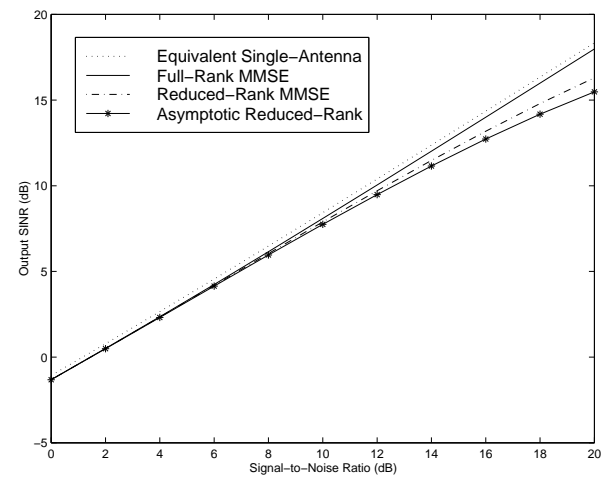

FIG. 5. Output SINR vs. input signal-tonoise ratio for MMSE receivers in line-of-sight transmission.

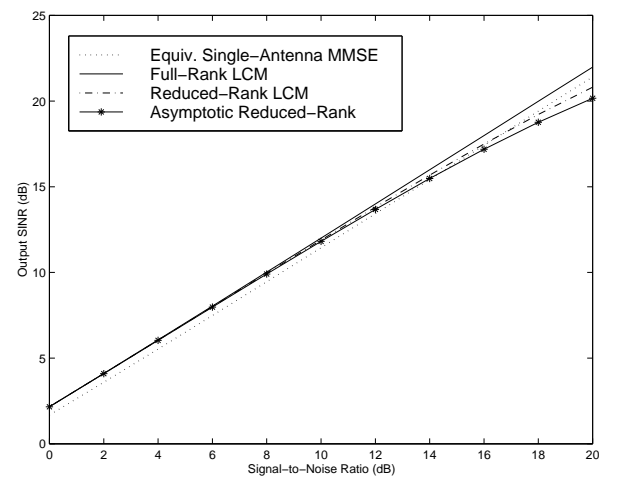

FIG. 6. Output SINR vs. input signal-tonoise ratio for LCM receivers in line-of-sight transmission.

can be directly applied here.

Figure 6 illustrates the performance of the LCM receivers in line-of- sight transmission. Again we see that Figure 6 resembles Figure 4. This agrees with our analytical result that their performances are asymptotically equal. Observations in Figure 4 can again be applied here.

6. Conclusion. In this paper, we obtain explicit expressions for the asymptotic eigenvalue moments of several classes of infinite random matrices that arise in multiuser detection in DS-CDMA system with random spreading. The results are used in the design of the asymptotic reduced-rank MMSE/LCM receivers that use the asymptotic values of the optimal weights. Numerical results show that the penalty in the output SINR due to using the asymptotic weights in place of the exact optimal weights is about $1 \mathrm{~dB}$ for reasonably large processing gain, e.g. 32. For larger processing gain, say 80 or larger, the performance loss is less than $0.5 \mathrm{~dB}$. The asymptotic reduced-rank receivers are desirable in real-time implementation of systems using long sequences because they do not depend on realizations of the spreading sequences. The equivalence of the asymptotic moments in these cases is used to obtain the asymptotic equality of the output SINR's of these receivers to that of the single antenna MMSE receiver with enlarged processing gain and possibly reduced noise level. The asymptotic equality is confirmed by numerical results. An explicit expression for the free cumulants of the asymptotic eigenvalues distribution of several classes of infinite random matrices is given from which the Tse-Hanly formula is proved. The technique used for finding the free cumulants of our infinite random matrices, has independent interest: in fact it permits, in the more general case, to find, using the free cumulants property of some suitable random matrix [19], an explicit expression for the moments of random matrices with more complex structure. 


\section{Appendix.}

A Proof of Proposition 1. Suppose that

$$
\mathbf{s}_{k}=\frac{1}{\sqrt{N}} \cdot\left(V_{k, 1}, \ldots, V_{k, N}\right)^{T},
$$

where $V_{k, j}$ 's are i.i.d. random variables that take equiprobable values on $\{ \pm 1\}$. Then

$$
\begin{aligned}
& \operatorname{Trace}\left\{\left(\mathbf{S D S}^{H}\right)^{m}\right\} \\
= & \operatorname{Trace}\left\{\left(\sum_{i_{1}=1}^{K} P_{i_{1}} \mathbf{s}_{i_{1}} \mathbf{s}_{i_{1}}^{H}\right) \cdots\left(\sum_{i_{m}=1}^{K} P_{i_{m}} \mathbf{s}_{i_{m}} \mathbf{s}_{i_{m}}^{H}\right)\right\} \\
= & \operatorname{Trace}\left\{\sum_{i_{1}, \ldots, i_{m}=1}^{K} P_{i_{1}} \cdots P_{i_{m}} \cdot \mathbf{s}_{i_{1}} \mathbf{s}_{i_{1}}^{H} \cdots \mathbf{s}_{i_{m}} \mathbf{s}_{i_{m}}^{H}\right\} \\
= & \operatorname{Trace}\left\{\sum_{i_{1}, \ldots, i_{m}=1}^{K} P_{i_{1}} \cdots P_{i_{m}} \cdot \mathbf{s}_{i_{m}}^{H} \mathbf{s}_{i_{1}} \cdots \mathbf{s}_{i_{m}-1}^{H} \mathbf{s}_{i_{m}}\right\} \\
= & \frac{1}{N^{m}} \sum_{i_{1}, \ldots, i_{m}=1}^{K} \sum_{j_{1}, \ldots, j_{m}=1}^{N} P_{i_{1}} \cdots P_{i_{m}} \cdot V_{i_{m}, j_{1}}^{*} V_{i_{1}, j_{1}} \cdots V_{i_{m-1}, j_{m}}^{*} V_{i_{m}, j_{m}} .
\end{aligned}
$$

where $P_{i}=A_{i}^{2}$. So,

$$
\text { (87) }=\frac{1}{N^{m+1}} \sum_{i_{1}, \ldots, i_{m}=1}^{K} \sum_{j_{1}, \ldots, j_{m}=1}^{N} P_{i_{1}} \cdots P_{i_{m}} \cdot \mathrm{E}\left[V_{i_{m}, j_{1}}^{*} V_{i_{1}, j_{1}} \cdots V_{i_{m-1}, j_{m}}^{*} V_{i_{m}, j_{m}}\right] \text {. }
$$

We then investigate all the possible ways to partition indices $i_{1}, \ldots, i_{m}$ into $k$ disjoint sets for $1 \leq k \leq m$. Indices in the same set keep identical in the summation and indices in different sets keep distinct in the summation. Suppose for a given partition of $i_{1}, \ldots, i_{m}$ into $k$ disjoint sets such that numbers of elements in the sets are $m_{1}, \ldots, m_{k}$, then this partition contributes a non-zero term $\mathrm{E}\left\{\Lambda^{m_{1}}\right\} \cdots \mathrm{E}\left\{\Lambda^{m_{k}}\right\} \cdot \beta^{k}$ to $\int \lambda^{m} d G(\lambda)$ if there exists a partition of $j_{1}, \ldots, j_{m}$ into $m-k+1$ disjoint sets such that in the following figure, every ordered pair in the upper row is matched with exactly one ordered pair in the lower row, and every ordered pair in the lower row is matched with exactly one ordered pair in the upper row.

$$
\begin{array}{llll}
\left(i_{1}, j_{1}\right) & \left(i_{2}, j_{2}\right) & \ldots & \left(i_{m}, j_{m}\right) \\
\left(i_{1}, j_{2}\right) & \left(i_{2}, j_{3}\right) & \ldots & \left(i_{m}, j_{1}\right)
\end{array}
$$

Two ordered pairs are matched iff the first indices in both of them are in the same set, and so are the second indices. The matching above ensures that no random variable 
appears alone in the expectation, which would make the expectation zero. If such a partition of $j_{1}, \ldots, j_{m}$ exists, we call it a good partition for the given partition of $i_{1}, \ldots, i_{m}$. Therefore, from the discussion above, $\int \lambda^{m} d G(\lambda)$ is the sum of all the terms

$$
c\left(m_{1}, \ldots, m_{k}\right) \cdot \mathrm{E}\left[\Lambda^{m_{1}}\right] \cdots \mathrm{E}\left[\Lambda^{m_{k}}\right] \cdot \beta^{k}
$$

for all $m_{1}+\cdots+m_{k}=m$ and all $1 \leq k \leq m$. The coefficient $c\left(m_{1}, \ldots, m_{k}\right)$ is the solution to the following combinatorial problem:

TheOREM 1. Among all the partitions of $i_{1}, \ldots, i_{m}$ into $k$ disjoint sets such that the cardinalities of these sets are $m_{1}, \ldots, m_{k}$ (totally there are $\frac{m !}{m_{1} ! \cdots m_{k} ! \cdot f\left(m_{1}, \ldots, m_{k}\right)}$ such partitions, where $f\left(m_{1}, \ldots, m_{k}\right)$ is defined in Section 2), the coefficient $c\left(m_{1}, \ldots\right.$, $m_{k}$ ) in (88) is the number of partitions such that there exists a good partition of $j_{1}, \ldots, j_{m}$ into $m-k+1$ disjoint sets for each of them.

Another combinatorial problem the solution of which is $c\left(m_{1}, \ldots, m_{k}\right)$ is proposed in [20], basically it is:

THEOREM 2. Consider $m$ balls arranged on a circle, numbered from 1 to $m$ clockwise. Among all the partitions of the $m$ balls into $k$ disjoint sets, such that the cardinalities of these sets are $m_{1}, \ldots, m_{k}$, the coefficient $c\left(m_{1}, \ldots, m_{k}\right)$ in (88) is the number of non-crossing partitions. A partition is called crossing if there exist two sets $A$ and $B$ and for some $a, b \in A$ and $c, d \in B$ such that the segment drawn from a to $b$ and the segment drawn from $c$ to $d$ cross.

We use Theorem 2 to obtain $c\left(m_{1}, \ldots, m_{k}\right)$, while the concept of mutually good partitions defined in Theorem 1 is used in the proof of extensions to Proposition 1. In what follows, we prove that

$$
c\left(m_{1}, \ldots, m_{k}\right)=\frac{m !}{(m-k+1) ! \cdot f\left(m_{1}, \ldots, m_{k}\right)} .
$$

In Theorem 2, sets that contain the same number of elements are considered indistinguishable. That is to say, if for some partition two sets (denoted by $A$ and $B$ ) both contain $p$ balls, then we consider the partition obtained by switching balls in $A$ and $B$ the same as the original one.

Here we first consider the case in which these sets are distinguishable and prove that the number of non-crossing partitions is given by

$$
\frac{m !}{(m-k+1) !}
$$


To make the sets distinguishable, we number them from 1 to $k$. The caridinality of $S_{i}$ is $m_{i}(1 \leq i \leq k)$. Now we select a ball, denoted by $b_{1}$, from the $m$ balls, then we select another ball, denoted by $b_{2}$, from the remaining $m-1$ balls, and so on until we select $b_{k-1}$. It is easy to see that there are $m ! /(m-k+1)$ ! ways to make the selection. Next, we describe a procedure that generates a non-crossing partition from any such selection. Then we prove that non-crossing partitions generated from different selections must also be different, and for each non-crossing partition, there must exist a selection that generates it.

(i) Suppose $b_{1}, \ldots, b_{k-1}$ are selected as in Figure 7 (without loss of generality, we assume that $b_{2}$ is located after $b_{1}$ on the circle, and so on.) Because $m_{1}+\cdots+m_{k}=m$, there must exist $i$ such that there are at least $m_{i}$ balls between $b_{i}$ and $b_{i+1}$, with $b_{i}$ included and $b_{i+1}$ excluded. We assign the $m_{i}$ balls starting from $b_{i}$ ( $b_{i}$ included) to set $S_{i}$. Then we take away the already assigned $m_{i}$ balls from the circle. Now we have a smaller circle of $m-m_{i}$ balls with $k-2$ selected. Then for the same reason, there exists $j$ such that there are at least $m_{j}$ balls between $b_{j}$ and the next selected ball after $b_{j}$, with $b_{j}$ included and the next selected ball after $b_{j}$ excluded. We then assign $m_{j}$ balls starting from $b_{j}$ to set $S_{j}$. Then the already assigned $m_{j}$ balls are taken away from the circle. This procedure can be repeated until the $k-1$ selected balls are used up. At that stage, we have $m-m_{1}-\cdots-m_{k-1}=m_{k}$ balls left, and we assign them to set $S_{k}$.

Since at each stage of the procedure described above, we always assign a continuous block of balls to a set, it is readily shown by induction that a partition generated by this procedure is non-crossing.

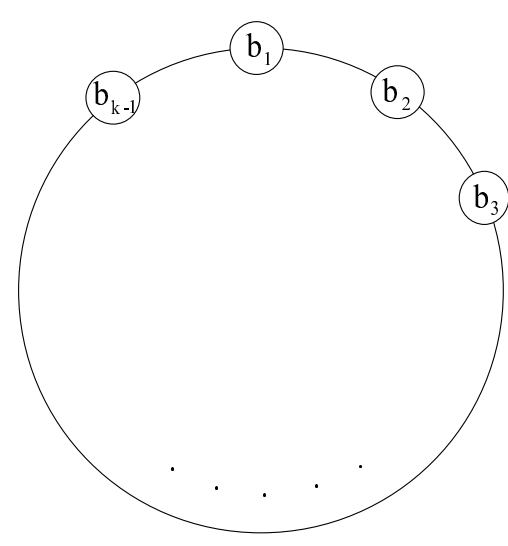

FIG. 7.

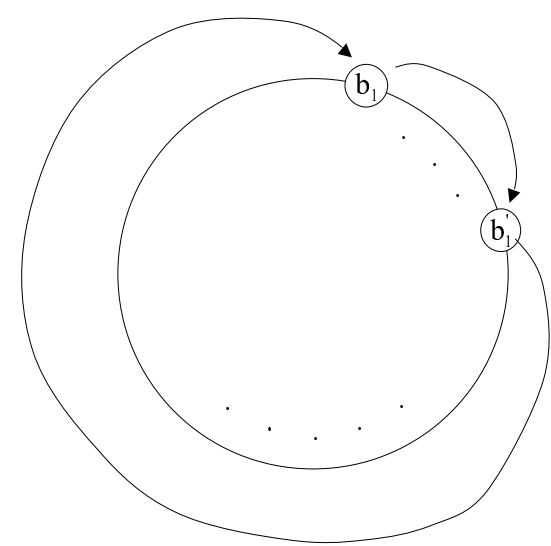

FIG. 8.

(ii) Here we prove that two different selections of balls, say $b_{1}, \ldots, b_{k-1}$ and $b_{1}^{\prime}, \ldots, b_{k-1}^{\prime}$, generate different partitions. We prove this by means of contradiction. Suppose that $b_{i}$ and $b_{i}^{\prime}$ are different shown as in Figure 8, and the two selections generate the same partition. Now we consider the balls assigned to set $S_{i}$ on the 
original circle of $m$ balls. According to the procedure in (i), we know that on the original circle, the $m_{i}$ balls in $S_{i}$ consist of several continuous blocks of balls, and both $b_{i}$ and $b_{i}^{\prime}$ must be the first (in clockwise direction) ball of the block they belong to. From the procedure in (i), for the first set of selected balls, we go clockwise from $b_{i}$ when we assign balls to $S_{i}$. Since $b_{i}^{\prime} \in S_{i}, b_{i}^{\prime}$ is reached. Similarly, for the second set of selected balls, $b_{i}$ is reached as we go clockwise from $b_{i}^{\prime}$ to assign balls to $S_{i}$. So, at the stage that we assign balls to $S_{i}$, we go clockwise from $b_{i}$ and returned to $b_{i}$ again (shown in Figure 8). That means, all the unassigned balls are traversed and assigned to $S_{i}$. However, this is a contradiction because after all the selected balls are used up, we still have to have $m_{k}$ unassigned balls left for $S_{k}$. Therefore we proved that different selections generate different partitions.

(iii) Here we prove that for every non-crossing partition, there exists a set of $k-1$ selected balls that generates it. First we introduce the following lemma which is proved at the end of Appendix A.

Lemma. For a given non-crossing partition, there exists a set $S_{i}$ such that all the balls in $S_{i}$ form a continuous block on the original circle.

Suppose that $S_{i}$ is such a set, we select the first ball (in clockwise direction) of $S_{i}$ as $b_{i}$. Then we take away $S_{i}$ from the circle. Now we have a partition of $m-m_{i}$ balls into $k-1$ disjoint sets which is non- crossing. The lemma above can be applied again. The same procedure can be repeated until $k-1$ balls are selected. It is easy to verify that this set of selected balls indeed generates the same partition that we start with.

From the discussion in (i), (ii), and (iii), a one-to-one and onto mapping from the selections of $k-1$ balls to the non-crossing partitions has been established. Therefore, the number of non-crossing partitions is given by

$$
\frac{m !}{(m-k+1) !}
$$

when sets are distinguishable.

If instead, sets are considered indistinguishable, the number of non-crossing partitions will be reduced. This is because we are free to switch balls in sets that contain the same number of balls and still get the same partition. For example, the partition does not change no matter $S_{1}=\{a, b\}$ and $S_{2}=\{c, d\}$ or $S_{2}=\{a, b\}$ and $S_{1}=\{c, d\}$. It is readily shown the number of non-crossing partitions is reduced by a factor $f\left(m_{1}, \ldots, m_{k}\right)$ and is given by

$$
\frac{m !}{(m-k+1) ! \cdot f\left(m_{1}, \ldots, m_{k}\right)} \text {. }
$$

The factor $f\left(m_{1}, \ldots, m_{k}\right)$ makes the sets of the same size indistinguishable by mixing up these sets. 
Proof of the Lemma. We pick up any set from the partition, if it consists a single continuous block (such a set is called a continuous set), the proof ends. If it consists of at least two separate continuous blocks, we pick up any two of them (denoted by Block 1 and Block 2, shown as in Figure 9). If there is a continuous set between $x_{1}$ and $x_{2}$ (with $x_{1}$ and $x_{2}$ included), the proof ends. If none of the sets between $x_{1}$ and $x_{2}$ is continuous, we pick up any set between $x_{1}$ and $x_{2}$ and consider any two blocks that belong to this set (denoted by Block 3 and Block 4, as shown in Figure 10). The same reasoning can be applied when we further consider sets between $y_{1}$ and $y_{2}$. This procedure must stop after finite number of steps. A continuous set is found when the procedure stops. The lemma is therefore proved.

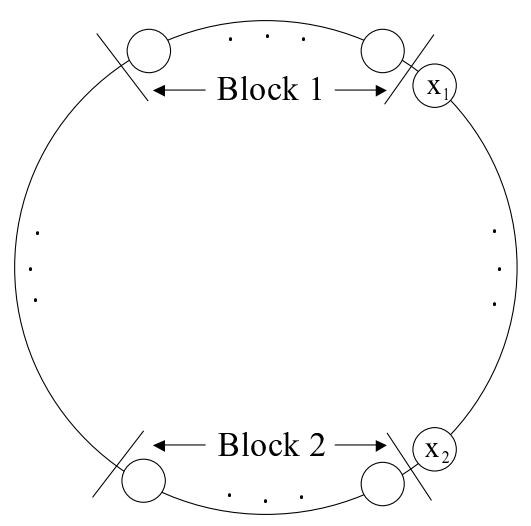

FIG. 9.

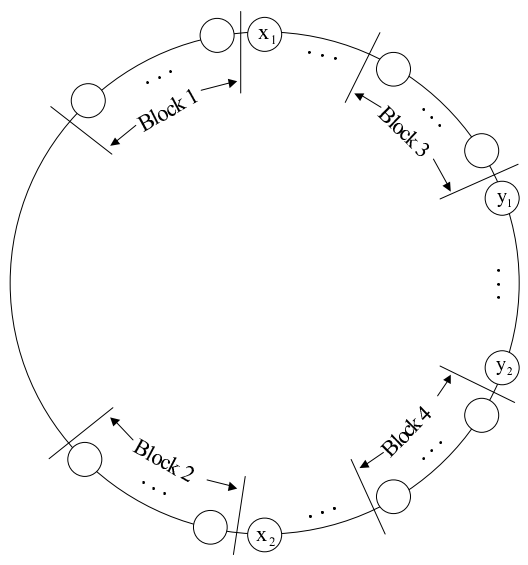

FIG. 10.

B Proof of Proposition 4. For simplicity of notation, we prove Proposition 4 only for the equal received power case. That is, $\mathbf{D}$ is assumed to be an identity matrix. The proof in the general unequal received power case can be obtained by incorporating the corresponding treatment in the proof for Proposition 1. As in the proof of Proposition 1,

$$
\int \lambda^{m} G(\lambda) d \lambda=\lim _{\substack{N, K \rightarrow \infty \\ \frac{K}{N}=\beta}} \frac{1}{N} \cdot \mathrm{E}\left[\operatorname{Trace}\left\{\left(\mathbf{S S}^{H}\right)^{m}\right\}\right]
$$

where $G(\lambda)$ is the limit eigenvalue distribution of $\mathbf{S S}^{H}$ as $K, N$ go to infinity with $K / N=\beta$. Denote

$$
\mathbf{s}_{k}=\frac{1}{\sqrt{N}} \cdot\left(V_{k, 1}, \ldots, V_{k, N}\right)^{T}
$$

then 
$(95)$

$$
\begin{aligned}
& \operatorname{Trace}\left\{\left(\mathbf{S S}^{H}\right)^{m}\right\} \\
= & \operatorname{Trace}\left\{\left(\sum_{i_{1}=1}^{K} \mathbf{s}_{i_{1}} \mathbf{s}_{i_{1}}^{H}\right) \cdots\left(\sum_{i_{m}=1}^{K} \mathbf{s}_{i_{m}} \mathbf{s}_{i_{m}}^{H}\right)\right\} \\
= & \operatorname{Trace}\left\{\sum_{i_{1}, \ldots, i_{m}=1}^{K} \mathbf{s}_{i_{1}} \mathbf{s}_{i_{1}}^{H} \cdots \mathbf{s}_{i_{m}} \mathbf{s}_{i_{m}}^{H}\right\} \\
= & \operatorname{Trace}\left\{\sum_{i_{1}, \ldots, i_{m}=1}^{K} \mathbf{s}_{i_{m}}^{H} \mathbf{s}_{i_{1}} \cdots \mathbf{s}_{i_{m-1}}^{H} \mathbf{s}_{i_{m}}\right\} \\
= & \frac{1}{N^{m}} \sum_{i_{1}, \ldots, i_{m}=1}^{K} \sum_{j_{1}, \ldots, j_{m}=1}^{N} V_{i_{m}, j_{1}}^{*} V_{i_{1}, j_{1}} \cdots V_{i_{m-1}, j_{m}}^{*} V_{i_{m}, j_{m}} .
\end{aligned}
$$

So,

$$
\begin{aligned}
& \frac{1}{N} \cdot \mathrm{E}\left[\operatorname{Trace}\left\{\left(\mathbf{S S}^{H}\right)^{m}\right\}\right] \\
= & \frac{1}{N^{m+1}} \sum_{i_{1}, \ldots, i_{m}=1}^{K} \sum_{j_{1}, \ldots, j_{m}=1}^{N} \mathrm{E}\left[V_{i_{m}, j_{1}}^{*} V_{i_{1}, j_{1}} \cdots V_{i_{m-1}, j_{m}}^{*} V_{i_{m}, j_{m}}\right] .
\end{aligned}
$$

Now for a given partition of $i_{1}, \ldots, i_{m}$ into $k(1 \leq k \leq m)$ disjoint sets, if there exists a good partition of $j_{1}, \ldots, j_{m}$ into $m-k+1$ disjoint sets, then

$$
V_{i_{m}, j_{1}}^{*} V_{i_{1}, j_{1}} \cdots V_{i_{m-1}, j_{m}}^{*} V_{i_{m}, j_{m}}
$$

is made into the product of $m$ modulo squares of random variables. Since the modulo square of each random variable is 1 , we get

$$
\mathrm{E}\left[V_{i_{m}, j_{1}}^{*} V_{i_{1}, j_{1}} \cdots V_{i_{m-1}, j_{m}}^{*} V_{i_{m}, j_{m}}\right]=1
$$

Eq. (103) holds for every $2 m$-tuple that $\left(i_{1}, \ldots, i_{m}, j_{1}, \ldots, j_{m}\right)$ takes values on. Let us recall that indices in the same set keep identical and indices in different sets keep distinct in the summation $\sum_{i_{1}, \ldots, i_{m}=1}^{K} \sum_{j_{1}, \ldots, j_{m}=1}^{N}$, so there are totally

$$
\frac{1}{N^{m+1}} \cdot K \cdots(K-k+1) \cdot N \cdots(N-m+k)
$$

$2 m$-tuples that $\left(i_{1}, \ldots, i_{m}, j_{1}, \ldots, j_{m}\right)$ take values on. Since each of them contribute 1 , the total contribution of the two mutually good partitions is (104). And the limit is 


$$
\lim _{\substack{N, K \rightarrow \infty \\ \frac{K}{N}=\beta}} \frac{1}{N^{m+1}} \cdot K \cdots(K-k+1) \cdot N \cdots(N-m+k)=\beta^{k} .
$$

From the proof of Proposition 1, we know that the two given mutually good partitions also contribute $\beta^{k}$ under the i.i.d. assumption there. Also from the proof of Proposition 1, we know that all non-mutually-good partitions contribute zero to

$$
\frac{1}{N} \cdot \mathrm{E}\left[\operatorname{Trace}\left\{\left(\mathbf{S S}^{H}\right)^{m}\right\}\right] .
$$

In what follows, we prove that this again holds true (at least asymptotically) here.

Suppose the partition of $i_{1}, \ldots, i_{m}$ into $k$ disjoint sets and the partition of $j_{1}, \ldots$, $j_{m}$ into $m-k+1$ disjoint sets are not mutually good. Then there is some factor in

$$
V_{i_{m}, j_{1}}^{*} V_{i_{1}, j_{1}} \cdots V_{i_{m-1}, j_{m}}^{*} V_{i_{m}, j_{m}}
$$

that appears alone. Without loss of generality, we assume it is $V_{i_{1}, j_{1}}$. This means, for every other factor in the product, $V_{i_{m-1}, j_{m}}^{*}$ for example, either $i_{m-1}$ and $i_{1}$ are not in the same set, or $j_{m}$ and $j_{1}$ are not in the same set.

For the given partition of $i_{1}, \ldots, i_{m}$, suppose that $i_{u}(1 \leq u \leq m)$ is in the same set as $i_{1}$ (this means $i_{u}$ and $i_{1}$ keep identical in the summation). We consider factors $V_{i_{u}, j_{u}}$ and $V_{i_{u}, j_{u+1}}^{*}$. Since $V_{i_{1}, j_{1}}$ is left unmatched and $i_{1}$ and $i_{u}$ are in the same set, $j_{1}$ and $j_{u}$ must belong to different sets (this means $j_{1}$ and $j_{u}$ keep distinct in the summation), and so must $j_{1}$ and $j_{u+1}$ belong to different sets. There are two cases. Case (i):

(i.a) For $V_{i_{u}, j_{u}}$, then $V_{i_{u}, j_{u}}=V_{i_{1}, j_{1}}^{*}$ only happens when $j_{u}=j_{1}+L \cdot N^{\prime}$ (when $\left.j_{1} \leq L \cdot N^{\prime}\right)$ or $j_{u}=j_{1}-L \cdot N^{\prime}$ (when $j_{1}>L \cdot N^{\prime}$ ). Restricting that $j_{u}=j_{1} \pm L \cdot N^{\prime}$ significantly reduces the total number of $2 m$-tuples that $\left(i_{1}, \ldots, i_{m}, j_{1}, \ldots, j_{m}\right)$ take values on. For example, if we only require that $j_{u} \neq j_{1}$, then the number of 2 -tuples that $\left(j_{1}, j_{u}\right)$ take values on is $N(N-1)$. However, if we restrict that $j_{u}=j_{1} \pm L \cdot N^{\prime}$, then the number of 2-tuples that $\left(j_{1}, j_{u}\right)$ take values on is $N$. Therefore, the number of $2 m$-tuples is not large enough to survive the $1 / N^{m+1}$ in (101) when we take the limit.

(i.b) Then we consider the case such that $V_{i_{u}, j_{u}} \neq V_{i_{1}, j_{1}}^{*}$. In this case, either (1) the random phase of $V_{i_{1}, j_{1}}$ is not going to be cancelled, or (2) the random chip value of $V_{i_{1}, j_{1}}$ is not going to be made into square. Since they are independent of everything else, the expectation

$$
\mathrm{E}\left[V_{i_{m}, j_{1}}^{*} V_{i_{1}, j_{1}} \cdots V_{i_{m-1}, j_{m}}^{*} V_{i_{m}, j_{m}}\right]
$$

can be decomposed into the product of the expectation of the random phase or the random chip value of $V_{i_{1}, j_{1}}$ and the expectation of everything else. 
From the fact that the expectation of the random phase and the random chip value is zero, we get

$$
\mathrm{E}\left[V_{i_{m}, j_{1}}^{*} V_{i_{1}, j_{1}} \cdots V_{i_{m-1}, j_{m}}^{*} V_{i_{m}, j_{m}}\right]=0
$$

Case (ii):

(ii.a) For $V_{i_{u}, j_{u+1}}^{*}$, then $V_{i_{u}, j_{u+1}}^{*}=V_{i_{1}, j_{1}}^{*}$ only happens when $j_{u+1}=j_{1}$. But this is not possible because $j_{u+1}$ and $j_{1}$ are always distinct.

(ii.b) Then we consider the case that $V_{i_{u}, j_{u+1}}^{*} \neq V_{i_{1}, j_{1}}^{*}$. The discussion is similar to (i.b).

Therefore from (i) and (ii), we know that contribution from non-mutually-good partitions is again always zero, and Proposition 4 is proved.

The proofs of Propositions 2, 3, 5 and 6 can be obtained following methods similar to those used in the proof of Proposition 4. Indeed, we notice that the classes of the matrices defined Proposition 2, 3, 5 and 6 can be considered as special cases of that defined in Proposition 4.

In Proposition 2, the matrix $\mathbf{S}$ with columns given by (3) is just a sub-block of the matrix in Proposition 4 obtained by considering only the first $L N^{\prime}$ first rows. And all discussion regarding the mutually good partitions in the proof of Proposition 4 can be repeated for Proposition 2; the difference between the two is the non-mutually-good partition. In Proposition 2, $V_{i_{u}, j_{u}}=V_{i_{1}, j_{1}}^{*}$ as discussed in case (i.a) will not happen. So according to (i.b) we have that:

$$
\mathrm{E}\left[V_{i_{m}, j_{1}}^{*} V_{i_{1}, j_{1}} \cdots V_{i_{m-1}, j_{m}}^{*} V_{i_{m}, j_{m}}\right]=0 .
$$

The discussion in Case (ii) still holds true.

Regarding Proposition 3, it is simply a special case of Proposition 4 without sequence repetitions.

Regarding Propositions 5 and 6 , we notice that the only difference between them and Propositions 2 and 4 is that in Propositions 5 and 6 the repeated sequences are multiplied by phases that are integer multiples of one fundamental uniformly distributed phase $\phi_{k}$ on $[0,2 \pi)$ (see Eq. (6) and Eq. (7)), while in Proposition 2 and 4 they are multiplied by independent and uniformly distributed phases $\left\{\phi_{k, l}\right\}_{l=1}^{L}$ (see Eq. (3), and Eq.(5)). According to the distribution of $\phi_{k}$, and from the fact that in the computation of the moments of the matrices only the average of mixed products of the entries of the matrices is involved, the discussion of the Case (i) and (ii) is again applicable.

C Proof of Equation 15. To begin we use Corollary 1 in [4] to show that as $N, K \rightarrow \infty$ while $K / N=\beta$ is kept constant:

$$
\mathbf{s}_{1}^{H}\left(\mathbf{S}_{1} \mathbf{D}_{1} \mathbf{S}_{1}^{H}\right)^{m} \mathbf{s}_{1}-\frac{1}{N} \operatorname{Trace}\left\{\left(\mathbf{S}_{1} \mathbf{D}_{1} \mathbf{S}_{1}^{H}\right)^{m}\right\} \stackrel{\text { a.s. }}{\rightarrow} 0
$$


To apply Corollary 1 in [4] we have condition on $\mathbf{S}_{1} \mathbf{D}_{1} \mathbf{S}_{1}^{H}$ and we use the fact that $\mathbf{s}_{1}$ and $\mathbf{S}_{1} \mathbf{D}_{1} \mathbf{S}_{1}^{H}$ are mutually statistically independent.

Next, observing that the empirical distribution of the diagonal elements of the matrix $\mathbf{D}_{1}$ converges almost surely (a.s.) to a fixed non-random limit distribution, we apply the results given in [21]:

$$
\frac{1}{N} \operatorname{Trace}\left\{\left(\mathbf{S}_{1} \mathbf{D}_{1} \mathbf{S}_{1}^{H}\right)^{m}\right\} \stackrel{a . s .}{\rightarrow} \lim _{\substack{N, K \rightarrow \infty \\ K=\beta}} \frac{1}{N} \mathrm{E}\left[\operatorname{Trace}\left\{\left(\mathbf{S}_{1} \mathbf{D}_{1} \mathbf{S}_{1}^{H}\right)^{m}\right\}\right]
$$

Then we have established that: $\mathbf{s}_{1}^{H}\left(\mathbf{S}_{1} \mathbf{D}_{1} \mathbf{S}_{1}^{H}\right)^{m} \mathbf{s}_{1}$ converges almost surely to:

$$
\frac{1}{N} \mathrm{E}\left[\operatorname{Trace}\left\{\left(\mathbf{S}_{1} \mathbf{D}_{1} \mathbf{S}_{1}^{H}\right)^{m}\right\}\right]=\int \lambda^{m} d G(\lambda) .
$$

\section{REFERENCES}

[1] G. E. Andrews, The Theory of Partitions. Cambridge Mathematical Library, Cambridge, 1984.

[2] K. Dyкema, On Certain Free Product via Extended Matrix Model, Journal of Functional Analysis, 112(1993), pp. 31-60.

[3] J.L. Doob, Stochastic Process, Wiley Classic Library Edition, New York, 1990.

[4] J.S. Evans AND D. TSE, Linear Multiuser Receivers for Multipath Fading Channels, IEEE Transactions on Information Theory, 46:6(2000), pp. 2059-2078.

[5] V.L. GIRKo, Theory of random determinants, Kluwer Academic Publishers, Boston 1990.

[6] I.S. Gradshteyn And I.M. RyzhiK, Table of Integrals, Series, and Products, Academic Press, New York, 1980.

[7] A. J. Grant And P. D. AlexAnder, Random sequence multisets for synchronous code-division multiple- access channels, IEEE Transactions on Information Theory, 44:7(1998), pp. 28322836.

[8] S. V. HANly And D. N. C. Tse, Resource Pooling and Effective Bandwidths in CDMA Networks with Multiuser Receivers and Spatial Diversity, IEEE Transactions on Information Theory, 47:4(2001), pp. 1328-1351.

[9] F. Hiai And D. Petz, Asymptotic freeness almost everywhere for random matrices, Acta Sci. Math. (Szeged), 66(2000), pp. 801-826.

[10] M. L. Honig AND W. XIAO, Performance of reduced-rank linear interference suppression for DS- CDMA, IEEE Transactions on Information Theory, 47:5(2001), pp. 1928-1946.

[11] S. Moshavi, E. G. Kanterakis, And D.L. Schilling, Multistage linear receivers for DS-CDMA systems, International Journal of Wireless Information Networks, 39:1(1996), pp.1-17.

[12] R. R. MüLler And S. Verdú, Spectral Efficiency of Low-Complexity Multiuser Detectors, ISIT 2000, Sorrento, Italy, June 25-30, 2000.

[13] J. W. SILVERSTEIN AND Z. D. BAI, On the empirical distribution of eigenvalues of a class of large dimensional random matrices, J. Multivariate Anal., 54:2(1995), pp. 175-192.

[14] R. Speicher, Free Probability Theory and Non-Crossing Partitions, in unpublished Note for Lecture, at 39e Seminare Lotharingien de Combinatoire.

[15] D. N. C. Tse And S. V. HANLy, Linear Multiuser Receivers: Effective Interference, Effective Bandwidth and User Capacity, IEEE Transactions on Information Theory, 45:2(1999), pp.641-657.

[16] A. Tulino And S. Verdú, Improved Linear Receivers for BPSK-CDMA subject to Fading, IEEE Journal Selected Areas, August 2001. 
[17] S. Verdú AND S. ShAmAI, Spectral efficiency of CDMA with random spreading, IEEE Transactions on Information Theory, 45:2(1999), pp.622-640.

[18] D. Voiculescu, Lectures on free probability theory, M. Emery et al., Lectures on Probability Theory and Statistics : École d'Éte de Probabilités de Saint-Flour xxviii-1998, Lecture Notes in Mathematics, Springer-Verlag, 2000.

[19] D. V. Voiculescu, K. J. Dykema, And A. NicA, Free Random Variables, CRM Monograph Series, Volume 1, Providence, Rhode Island, USA: American Mathematical Society, 1992.

[20] W. XIAO AND M. L. HonIG, Convergence analysis of adaptive full-rank and multi-stage reducedrank interference suppression, 2000 Conference on Information Sciences and Systems, Princeton University, March 2000.

[21] Y. Q. YIN, Limiting spectral distribution for a class of random matrices, J. Multivariate Anal., 20(1986), pp. 50-68. 
